\title{
BMO FUNCTIONS AND BALAYAGE OF CARLESON MEASURES IN THE BESSEL SETTING
}

\author{
V. ALMEIDA, J. J. BETANCOR, A. J. CASTRO, J. C. FARIÑA, AND L. RODRÍGUEZ-MESA
}

\begin{abstract}
By $B M O_{\circ}(\mathbb{R})$ we denote the space consisting of all those odd and bounded mean oscillation functions on $\mathbb{R}$. In this paper we characterize the functions in $B M O_{\mathrm{o}}(\mathbb{R})$ with bounded support as those ones that can be written as a sum of a bounded function on $(0, \infty)$ plus the balayage of a Carleson measure on $(0, \infty) \times(0, \infty)$ with respect to the Poisson semigroup associated with the Bessel operator
\end{abstract}

$$
B_{\lambda}:=-x^{-\lambda} \frac{d}{d x} x^{2 \lambda} \frac{d}{d x} x^{-\lambda}, \quad \lambda>0 .
$$

This result can be seen as an extension to Bessel setting of a classical result due to Carleson.

\section{INTRODUCTION}

In this paper we extend [7, Theorem 2] (See also [29, Theorem A]) due to Carleson to Bessel settings.

A measurable function $f$ on $\mathbb{R}^{n}$ is said to have bounded mean oscillation, in short $f \in B M O\left(\mathbb{R}^{n}\right)$, when there exists $C>0$ such that, for every cube $Q \subset \mathbb{R}^{n}$ with sides parallel to the coordinate axes,

where

$$
\frac{1}{|Q|} \int_{Q}\left|f(y)-f_{Q}\right| d y \leq C,
$$

$$
f_{Q}:=\frac{1}{|Q|} \int_{Q} f(y) d y .
$$

It is defined, for every $f \in B M O\left(\mathbb{R}^{n}\right)$,

$$
\|f\|_{B M O\left(\mathbb{R}^{n}\right)}:=\sup _{Q} \frac{1}{|Q|} \int_{Q}\left|f(y)-f_{Q}\right| d y,
$$

where the supremum is taken over all the cubes $Q \subset \mathbb{R}^{n}$ with sides parallel to the coordinate axis. It is clear that $\|\cdot\|_{B M O\left(\mathbb{R}^{n}\right)}$ is a norm when two functions $f$ and $g$ in $B M O\left(\mathbb{R}^{n}\right)$ are identified provided that the difference $f-g$ is constant in $\mathbb{R}^{n} . B M O\left(\mathbb{R}^{n}\right)$ is also called the John-Nirenberg space $([18)$.

The $B M O\left(\mathbb{R}^{n}\right)$ space is closely connected with the so called Carleson measure in $\mathbb{R}_{+}^{n+1}:=$ $\mathbb{R}^{n} \times(0, \infty)$. If $Q$ is a cube in $\mathbb{R}^{n}$, the Carleson box is given by $\widehat{Q}:=Q \times(0,|Q|)$, where $|Q|$ denotes the Lebesgue measure of $Q$. A Borel measure $\mu$ on $\mathbb{R}_{+}^{n+1}$ is said to be a Carleson measure, in short $\mu \in \mathcal{C}\left(\mathbb{R}_{+}^{n+1}\right)$, when there exists $C>0$ such that, for every cube $Q \subset \mathbb{R}^{n}$ with sides parallel to the coordinate axes,

$$
\frac{|\mu|(\widehat{Q})}{|Q|} \leq C,
$$

where $|\mu|$ denotes the total variation measure of $\mu$.

If $\mu \in \mathcal{C}\left(\mathbb{R}_{+}^{n+1}\right)$ it can be defined the norm

$$
\|\mu\|_{\mathcal{C}\left(\mathbb{R}_{+}^{n+1}\right)}:=\sup _{Q} \frac{|\mu|(\widehat{Q})}{|Q|}
$$

where the supremum is taken over all cubes $Q \subset \mathbb{R}^{n}$ with sides parallel to the coordinate axes.

Last modification: April 17, 2018.

2010 Mathematics Subject Classification. 30H35, 35J15, 42B35, 42B37, 42C05.

Key words and phrases. Bessel operators, BMO functions, Carleson measure, balayage.

The authors are partially supported by MTM2016-79436-P. 
The classical Poisson semigroup $\left\{P_{t}\right\}_{t>0}$ generated by $-\sqrt{-\Delta}$, where $\Delta$ represents the Laplace operator $\Delta:=\sum_{i=1}^{n} \partial_{x_{i}}^{2}$ in $\mathbb{R}^{n}$, is defined for every $f \in L^{p}\left(\mathbb{R}^{n}\right), 1 \leq p \leq \infty$, by

$$
P_{t}(f)(x):=c_{n} \int_{\mathbb{R}^{n}} P_{t}(x-y) f(y) d y, \quad x \in \mathbb{R}^{n}, t>0,
$$

where $c_{n}:=\Gamma((n+1) / 2) / \pi^{(n+1) / 2}$. Here the Poisson kernel is

$$
P_{t}(x):=\frac{t}{\left(|x|^{2}+t^{2}\right)^{(n+1) / 2}}, \quad x \in \mathbb{R}^{n}, t>0 .
$$

If $f \in B M O\left(\mathbb{R}^{n}\right)$, then $([25$, p. 141])

$$
\int_{\mathbb{R}^{n}} \frac{|f(y)|}{(1+|y|)^{n+1}} d y<\infty
$$

and $P_{t}(|f|)(x)<\infty$, for every $x \in \mathbb{R}^{n}$ and $t>0$.

It is well known ([25, p. 159 and p. 165]) that a function $f \in L^{1}\left(\mathbb{R}^{n},(1+|y|)^{-n-1} d y\right)$ is in $B M O\left(\mathbb{R}^{n}\right)$ if, and only if, the measure $\mu_{f}$ on $\mathbb{R}_{+}^{n+1}$ defined by

$$
d \mu_{f}(x, t):=\left|t \partial_{t} P_{t}(f)(x)\right|^{2} \frac{d x d t}{t}, \quad(x, t) \in \mathbb{R}_{+}^{n+1},
$$

is a Carleson measure.

If $\mu$ is a positive measure on $\mathbb{R}_{+}^{n+1}$ the balayage $S_{\mu, P}$ with respect to the Poisson semigroup $\left\{P_{t}\right\}_{t>0}$ is defined by

$$
S_{\mu, P}(x):=\int_{\mathbb{R}_{+}^{n+1}} P_{t}(x-y) d \mu(y, t), \quad x \in \mathbb{R}^{n} .
$$

Carleson ([7, Theorem 2]) (see also [29, Theorem A]) proved that a function $f$ with compact support is in $B M O\left(\mathbb{R}^{n}\right)$ if, and only if, there exist $g \in L^{\infty}\left(\mathbb{R}^{n}\right)$ and a Carleson measure $\mu$ on $\mathbb{R}_{+}^{n+1}$ such that $f=g+S_{\mu, P}$ and

$$
\|f\|_{B M O\left(\mathbb{R}^{n}\right)} \sim\|g\|_{L^{\infty}\left(\mathbb{R}^{n}\right)}+\|\mu\|_{\mathcal{C}\left(\mathbb{R}_{+}^{n+1}\right)} .
$$

Actually, this result was established for uniparametric families $\left\{K_{t}\right\}_{t>0}$ being the Poisson semigroup $\left\{P_{t}\right\}_{t>0}$ a special case. An extension of [7, Theorem 2] to spaces of homogeneous type was proved by Uchiyama (27]). The proofs of the mentioned results in [7 and 27] (see also [17) are based on an iterative argument. Other proof was presented in 29. Here, we will adapt Wilson's ideas to our setting.

Recently, Chen, Duong, Li, Song and Yan ([] $]$ ) have established a version of Carleson's result ([7, Theorem 2]) where the Laplace operator $\Delta$ is replaced by the Schrödinger operator $\mathcal{L}_{V}:=$ $-\Delta+V$, where the nonnegative potential $V$ belongs to the reverse Hölder class $B_{q}$ for some $q \geq n$. Definitions and main properties about $B M O$ spaces associated with $\mathcal{L}_{V}$ can be encountered in 13 and 14 (see also 24]).

Harmonic analysis associated with Bessel operators was initiated by Muckenhoupt and Stein ([21]). They considered the Bessel operators

$$
\Delta_{\lambda}:=-x^{-2 \lambda} \frac{d}{d x} x^{2 \lambda} \frac{d}{d x}, \quad \lambda>0,
$$

and studied $L^{p}$-boundedness properties of maximal operators associated with Poisson semigroups defined by $\Delta_{\lambda}$ and Riesz transforms in this setting. Recently, harmonic analysis related to Bessel operators has raised interest again (see [2], [9, [10], 20], 22], 23], 28] and [30], among others).

We consider the Bessel type operator on $(0, \infty)$

$$
B_{\lambda}:=-x^{-\lambda} \frac{d}{d x} x^{2 \lambda} \frac{d}{d x} x^{-\lambda}=-\frac{d^{2}}{d x^{2}}+\frac{\lambda(\lambda-1)}{x^{2}}, \quad \lambda>0 .
$$

Note that the potential $V_{\lambda}(x):=\lambda(\lambda-1) / x^{2}, x \in(0, \infty)$, does not satisfy any reverse Hölder property and it has a singularity at $x=0$. Then, $B_{\lambda}$ is not included in the class of Schrödinger operators considered in 8 and [14].

Assume that $\lambda>0$. According to [21, $\S 16]$ the Poisson semigroup $\left\{P_{t}^{\lambda}\right\}_{t>0}$ associated with the Bessel operator $B_{\lambda}$ is defined as follows

$$
P_{t}^{\lambda}(f)(x):=\int_{0}^{\infty} P_{t}^{\lambda}(x, y) f(y) d y, \quad x, t \in(0, \infty)
$$


for every $f \in L^{p}(0, \infty), 1 \leq p \leq \infty$. Here, the Poisson kernel $P_{t}^{\lambda}(x, y)$ is given by

$$
P_{t}^{\lambda}(x, y):=\frac{2 \lambda}{\pi}(x y)^{\lambda} t \int_{0}^{\pi} \frac{(\sin \theta)^{2 \lambda-1}}{\left[(x-y)^{2}+t^{2}+2 x y(1-\cos \theta)\right]^{\lambda+1}} d \theta, \quad x, y, t \in(0, \infty) .
$$

For every $1 \leq p \leq \infty$, the family $\left\{P_{t}^{\lambda}\right\}_{t>0}$ is bounded on $L^{p}(0, \infty)$. Note that $\left\{P_{t}^{\lambda}\right\}_{t>0}$ is not Markovian, that is, $P_{t}^{\lambda}(1) \neq 1$. Indeed, according to [6, Lemma 2.2 and Remark 2.5] the function $v(x, t):=P_{t}^{\lambda}(1)(x)$ satisfies

$$
\left(\partial_{t}^{2}-B_{\lambda, x}\right) v(x, t)=0, \quad x, t \in(0, \infty)
$$

but clearly

$$
\left(\partial_{t}^{2}-B_{\lambda, x}\right) 1=-\frac{\lambda(\lambda-1)}{x^{2}}, \quad x, t \in(0, \infty) .
$$

We also remark that the function $\mathfrak{f}_{\lambda}(x)=x^{\lambda}, x \in(0, \infty)$, does not belong to $L^{p}(0, \infty)$, for any $1 \leq p \leq \infty$. However, $P_{t}^{\lambda}\left(\mathfrak{f}_{\lambda}\right)=\mathfrak{f}_{\lambda}, t>0$ (see, [1, p. 455]).

We denote by $P_{*}^{\lambda}$ the maximal operator defined by $\left\{P_{t}^{\lambda}\right\}_{t>0}$, that is,

$$
P_{*}^{\lambda}(f):=\sup _{t>0}\left|P_{t}^{\lambda}(f)\right|, \quad f \in L^{p}(0, \infty), \quad 1 \leq p \leq \infty .
$$

$P_{*}^{\lambda}$ is bounded from $L^{p}(0, \infty)$ into itself when $1<p \leq \infty$ and from $L^{1}(0, \infty)$ into $L^{1, \infty}(0, \infty)(\underline{6}$, Theorem 2.4 and Remark 2.5]).

The Hardy space $H_{\lambda}^{1}(0, \infty)$ associated to the operator $B_{\lambda}$ was studied in [5]. It is said that a function $f \in L^{1}(0, \infty)$ is in $H_{\lambda}^{1}(0, \infty)$ when $P_{*}^{\lambda}(f) \in L^{1}(0, \infty)$. On $H_{\lambda}^{1}(0, \infty)$ it is considered the norm $\|\cdot\|_{H_{\lambda}^{1}(0, \infty)}$ given by

$$
\|f\|_{H_{\lambda}^{1}(0, \infty)}:=\|f\|_{L^{1}(0, \infty)}+\left\|P_{*}^{\lambda}(f)\right\|_{L^{1}(0, \infty)}, \quad f \in H_{\lambda}^{1}(0, \infty) .
$$

The dual space of $H_{\lambda}^{1}(0, \infty)$ can be characterized as a $B M O$-type space. A function $f \in L^{1}(0, a)$, for every $a>0$, is in $B M O_{\mathrm{o}}(\mathbb{R})$ when there exists $C>0$ such that

a) for every bounded interval $I \subset(0, \infty)$,

$$
\frac{1}{|I|} \int_{I}\left|f(y)-f_{I}\right| d y \leq C,
$$

b) for every $a \in(0, \infty)$,

$$
\frac{1}{a} \int_{0}^{a}|f(y)| d y \leq C .
$$

On $B M O_{\circ}(\mathbb{R})$ the norm $\|\cdot\|_{B M O_{o}(\mathbb{R})}$ is defined by

$$
\left.\left.\|f\|_{B M O_{\mathrm{o}}(\mathbb{R})}:=\inf \{C>0: a) \text { and } b\right) \text { hold }\right\} .
$$

The space $B M O_{\mathrm{o}}(\mathbb{R})$ can be characterized as that one consisting on all the functions $f$ defined on $(0, \infty)$ such that the odd extension $f_{\mathrm{o}}$ of $f$ to $\mathbb{R}$ is in $B M O(\mathbb{R})$ ([3, p.465]). This property, that justifies the notation $B M O_{\mathrm{o}}(\mathbb{R})$ for our space, will be very useful in the sequel. The space $B M O_{\text {o }}(\mathbb{R})$ coincides, in the usual way, with the dual space of $H_{\lambda}^{1}(0, \infty)$ (see [3, p. 466]).

We say that a Borel measure $\mu$ on $(0, \infty) \times(0, \infty)$ is a Carleson measure on $(0, \infty) \times(0, \infty)$ when there exists $C>0$ such that, for every bounded interval $I \subset(0, \infty)$,

$$
\frac{|\mu|(\widehat{I})}{|I|} \leq C .
$$

Here, as above, $|\mu|$ represents the total variation measure of $\mu,|I|$ denotes the length of the interval $I$ and $\widehat{I}:=I \times(0,|I|)$. If $\mu$ is a Carleson measure on $(0, \infty) \times(0, \infty)$ we define

$$
\|\mu\|_{\mathcal{C}}:=\sup _{I} \frac{|\mu|(\widehat{I})}{|I|}
$$

where the supremum is taken over all bounded intervals $I \subset(0, \infty)$.

Next result shows the connection between $B M O_{\mathrm{o}}(\mathbb{R})$ and the Carleson measures on $(0, \infty) \times$ $(0, \infty)$ by using Poisson semigroups $\left\{P_{t}^{\lambda}\right\}_{t>0}$. 
Theorem A. ([3, Theorem 1.1]) Let $\lambda>0$. Suppose that $f \in L^{1}(0, a)$, for every a $>0$. Then, $f \in B M O_{o}(\mathbb{R})$ if, and only if, $f \in L^{1}\left((0, \infty),(1+x)^{-2} d x\right)$ and the measure $\mu_{f}$ on $(0, \infty) \times(0, \infty)$ defined by

$$
\mu_{f}(x, t):=\left|t \partial_{t} P_{t}^{\lambda}(f)(x)\right|^{2} \frac{d x d t}{t}, \quad x, t \in(0, \infty),
$$

is Carleson. Moreover, the quantities $\|f\|_{B M O_{o}(\mathbb{R})}^{2}$ and $\left\|\mu_{f}\right\|_{\mathcal{C}}$ are equivalent.

In our $B_{\lambda}$-Bessel setting we consider the gradient $\nabla_{\lambda}:=\left(\partial_{t}, D_{\lambda, x}\right)$, where $D_{\lambda, x}:=x^{\lambda} \partial_{x} x^{-\lambda}$.

Theorem B. ([1, Theorem 1]) Let $\lambda>1$. Assume that $u$ is a function defined in $\mathbb{R} \times(0, \infty)$ such that $x^{-\lambda} u(x, t) \in C^{\infty}(\mathbb{R} \times(0, \infty))$ and it is even in the $x$-variable. Suppose also that $\left(\partial_{t}^{2}-B_{\lambda}\right) u=0$, on $(0, \infty) \times(0, \infty)$. Then, the following assertions are equivalent.

(i) There exists $f \in B M O_{o}(\mathbb{R})$ such that $u(x, t)=P_{t}^{\lambda}(f)(x), x, t \in(0, \infty)$.

(ii) The measure $\mu_{\lambda}$ on $(0, \infty) \times(0, \infty)$ defined by

$$
d \mu_{\lambda}(x, t):=\left|t \nabla_{\lambda} u(x, t)\right|^{2} \frac{d x d t}{t}, \quad x, t \in(0, \infty)
$$

is Carleson.

Moreover, the quantities $\|f\|_{B M O_{o}(\mathbb{R})}^{2}$ and $\left\|\mu_{\lambda}\right\|_{\mathcal{C}}$ are equivalent.

The main result of this paper is the following, which can be seen as a version of the Carleson's result in [7, Theorem 2] (see also [29, Theorem A]) in our Bessel setting.

Theorem 1.1. Let $\lambda>0$.

(i) If $\mu$ is a Carleson measure on $(0, \infty) \times(0, \infty)$, the balayage of $\mu$ with respect to the Poisson semigroup $\left\{P_{t}^{\lambda}\right\}_{t>0}$ associated with $B_{\lambda}$ defined by

$$
S_{\mu, P^{\lambda}}(x):=\int_{0}^{\infty} \int_{0}^{\infty} P_{t}^{\lambda}(x, y) d \mu(y, t), \quad x \in(0, \infty)
$$

is in $B M O_{o}(\mathbb{R})$ and

$$
\left\|S_{\mu, P^{\lambda}}\right\|_{B M O_{o}(\mathbb{R})} \leq C\|\mu\|_{\mathcal{C}} .
$$

Here $C>0$ does not depend on $\mu$.

(ii) Let $f \in B M O_{o}(\mathbb{R})$ such that $f=0$ on $(a, \infty)$, for some $a>0$. Then, there exist $g \in$ $L^{\infty}(0, \infty)$ and a Carleson measure $\mu$ on $(0, \infty) \times(0, \infty)$ such that $f=g+S_{\mu, P^{\lambda}}$ and

$$
\|g\|_{L^{\infty}(0, \infty)}+\|\mu\|_{\mathcal{C}} \leq C\|f\|_{B M O_{o}(\mathbb{R})},
$$

where $C>0$ does not depend on $f$.

In order to prove this theorem we are going to adapt the procedure developed by Wilson ([29]) to our Bessel setting.

The heat semigroup $\left\{W_{t}^{\lambda}\right\}_{t>0}$ associated to the Bessel operator $B_{\lambda}$ is defined, for every $f \in$ $L^{p}(0, \infty), 1 \leq p \leq \infty$, by

$$
W_{t}^{\lambda}(f)(x):=\int_{0}^{\infty} W_{t}^{\lambda}(x, y) f(y) d y, \quad x \in(0, \infty),
$$

where the heat kernel is given by

$$
W_{t}^{\lambda}(x, y):=\frac{\sqrt{x y}}{2 t} I_{\lambda-1 / 2}\left(\frac{x y}{2 t}\right) e^{-\left(x^{2}+y^{2}\right) /(4 t)}, \quad x, y, t \in(0, \infty) .
$$

Here, $I_{\nu}$ denotes the modified Bessel function of the first kind and order $\nu$. If $\mu$ is a Borel measure on $(0, \infty) \times(0, \infty)$ we define the balayage $S_{\mu, W^{\lambda}}$ of $\mu$ with respect to $\left\{W_{t}^{\lambda}\right\}_{t>0}$ in the natural way.

The well known subordination formula connects Bessel Poisson and heat semigroups as follows. For every $f \in L^{p}(0, \infty), 1 \leq p \leq \infty$,

$$
P_{t}^{\lambda}(f)(x)=\frac{1}{\sqrt{\pi}} \int_{0}^{\infty} \frac{e^{-u}}{\sqrt{u}} W_{t^{2} /(4 u)}^{\lambda}(f)(x) d u, \quad x, t \in(0, \infty) .
$$

By using this equality from Theorem 1.1 we can immediately deduce the following property (see [8, proof of Theorem 3.5]). 
Corollary 1.2. Let $\lambda>0$ and $f \in B M O_{o}(\mathbb{R})$ such that $f=0$ on $(a, \infty)$, for some $a>0$. Then, there exist $g \in L^{\infty}(0, \infty)$ and a Carleson measure $\mu$ on $(0, \infty) \times(0, \infty)$ such that $f=g+S_{\mu, W^{\lambda}}$ and

Here $C>0$ does not depend on $f$.

$$
\|g\|_{L^{\infty}(0, \infty)}+\|\mu\|_{\mathcal{C}} \leq C\|f\|_{B M O_{o}(\mathbb{R})} .
$$

This paper is organized as follows. In Section 2, we present some properties of the Poisson kernel and Poisson semigroups for Bessel operators that will be useful in the sequel. In Section 3 we prove new properties of the space $B M O_{\circ}(\mathbb{R})$ that are needed to establish Theorem 1.1. The proof of Theorem 1.1 is presented in Section 4

Throughout this paper by $C$ we always denote a positive constant that is not necessarily the same in each occurrence. Also, we always consider $\lambda>0$.

\section{Some useful properties of Bessel Poisson semigroups}

As it was mentioned in the introduction, according to $[21, \S 16]$ the $B_{\lambda}$-Poisson kernel is given by

$$
P_{t}^{\lambda}(x, y):=\frac{2 \lambda}{\pi} t(x y)^{\lambda} \int_{0}^{\pi} \frac{(\sin \theta)^{2 \lambda-1}}{\left[(x-y)^{2}+t^{2}+2 x y(1-\cos \theta)\right]^{\lambda+1}} d \theta, \quad x, y, t \in(0, \infty) .
$$

From (2.1) it is straightforward that

$$
0 \leq P_{t}^{\lambda}(x, y) \leq C \frac{t(x y)^{\lambda}}{\left((x-y)^{2}+t^{2}\right)^{\lambda+1}}, \quad x, y, t \in(0, \infty) .
$$

Also, by [21, (b) p. 86] we get

$$
P_{t}^{\lambda}(x, y) \leq C \frac{t}{(x-y)^{2}+t^{2}}, \quad x, y, t \in(0, \infty) .
$$

We also need estimations for the derivatives of $P_{t}^{\lambda}(x, y)$.

Lemma 2.1. Let $\lambda>0$. Then, for every $t, x, y \in(0, \infty)$,

$$
\left|\partial_{t} P_{t}^{\lambda}(x, y)\right|+\left|D_{\lambda, x} P_{t}^{\lambda}(x, y)\right| \leq \frac{C}{t} P_{t}^{\lambda}(x, y),
$$

Proof. We have that

$$
\begin{aligned}
\left|\partial_{t} P_{t}^{\lambda}(x, y)\right| & =\mid \frac{2 \lambda}{\pi}(x y)^{\lambda}\left\{\int_{0}^{\pi} \frac{(\sin \theta)^{2 \lambda-1}}{\left[(x-y)^{2}+t^{2}+2 x y(1-\cos \theta)\right]^{\lambda+1}} d \theta\right. \\
& \left.-2(\lambda+1) t^{2} \int_{0}^{\pi} \frac{(\sin \theta)^{2 \lambda-1}}{\left[(x-y)^{2}+t^{2}+2 x y(1-\cos \theta)\right]^{\lambda+2}} d \theta\right\} \mid \\
& \leq \frac{C}{t} P_{t}^{\lambda}(x, y) .
\end{aligned}
$$

On the other hand, we can write

and also,

$$
D_{\lambda, x} P_{t}^{\lambda}(x, y)=-\frac{4 \lambda(\lambda+1)}{\pi} t(x y)^{\lambda} \int_{0}^{\pi} \frac{((x-y)+y(1-\cos \theta))(\sin \theta)^{2 \lambda-1}}{\left[(x-y)^{2}+t^{2}+2 x y(1-\cos \theta)\right]^{\lambda+2}} d \theta,
$$

$$
D_{\lambda, x} P_{t}^{\lambda}(x, y)=-\frac{4 \lambda(\lambda+1)}{\pi} t(x y)^{\lambda} \int_{0}^{\pi} \frac{((x-y) \cos \theta+x(1-\cos \theta))(\sin \theta)^{2 \lambda-1}}{\left((x-y)^{2}+t^{2}+2 x y(1-\cos \theta)\right)^{\lambda+2}} d \theta .
$$

Then,

$$
\left|D_{\lambda, x} P_{t}^{\lambda}(x, y)\right| \leq C t(x y)^{\lambda} \int_{0}^{\pi} \frac{(|x-y|+\min \{x, y\}(1-\cos \theta))(\sin \theta)^{2 \lambda-1}}{\left[(x-y)^{2}+t^{2}+2 x y(1-\cos \theta)\right]^{\lambda+2}} d \theta .
$$

Since

$$
\frac{|x-y|+\min \{x, y\}(1-\cos \theta)}{(x-y)^{2}+t^{2}+2 x y(1-\cos \theta)} \leq \frac{C}{t}\left(1+\frac{\min \{x, y\}}{\sqrt{x y}}\right) \leq \frac{C}{t}, \quad x, y, t \in(0, \infty) \text { and } \theta \in[0, \pi],
$$

we conclude that

$$
\left|D_{\lambda, x} P_{t}^{\lambda}(x, y)\right| \leq \frac{C}{t} P_{t}^{\lambda}(x, y)
$$


The Hankel transform $h_{\lambda}(f)$ of $f \in L^{1}(0, \infty)$ is defined by

$$
h_{\lambda}(f)(x):=\int_{0}^{\infty} \sqrt{x y} J_{\lambda-1 / 2}(x y) f(y) d y, \quad x \in(0, \infty),
$$

where $J_{\nu}$ denotes the Bessel function of the first kind and order $\nu$. Since the function $\sqrt{z} J_{\lambda-1 / 2}(z)$ is bounded on $(0, \infty)$, it is clear that

$$
\left\|h_{\lambda}(f)\right\|_{L^{\infty}(0, \infty)} \leq C\|f\|_{L^{1}(0, \infty)}, \quad f \in L^{1}(0, \infty) .
$$

The Hankel transform $h_{\lambda}$ can be extended from $L^{1}(0, \infty) \cap L^{2}(0, \infty)$ to $L^{2}(0, \infty)$ as an isometry in $L^{2}(0, \infty)([26$, p. $473(1)])$.

The Bessel Poisson kernel can be written in the following way ([21, (16.1')])

$$
P_{t}^{\lambda}(x, y)=\int_{0}^{\infty} e^{-t z} \sqrt{x z} J_{\lambda-1 / 2}(x z) \sqrt{y z} J_{\lambda-1 / 2}(y z) d z, \quad x, y, t \in(0, \infty) .
$$

Then, we have that

$$
P_{t}^{\lambda}(x, y)=h_{\lambda}\left(e^{-t z} \sqrt{x z} J_{\lambda-1 / 2}(x z)\right)(y), \quad x, y, t \in(0, \infty),
$$

and

$$
\partial_{t} P_{t}^{\lambda}(x, y)=-h_{\lambda}\left(z e^{-t z} \sqrt{x z} J_{\lambda-1 / 2}(x z)\right)(y), \quad x, y, t \in(0, \infty) .
$$

We can also obtain that if $f \in L^{2}(0, \infty)$,

$$
P_{t}^{\lambda}(f)=h_{\lambda}\left(e^{-t z} h_{\lambda}(f)(z)\right), \quad t \in(0, \infty),
$$

and

$$
\partial_{t} P_{t}^{\lambda}(f)=-h_{\lambda}\left(z e^{-t z} h_{\lambda}(f)(z)\right), \quad t \in(0, \infty) .
$$

Indeed, since the function $\sqrt{z} J_{\lambda}(z)$ is bounded on $(0, \infty)$, we get

$$
\begin{aligned}
& \int_{0}^{\infty}\left|\sqrt{y z} J_{\lambda-1 / 2}(y z) e^{-t z} h_{\lambda}(f)(z)\right| d z \leq C \int_{0}^{\infty} e^{-t z}\left|h_{\lambda}(f)(z)\right| d z \\
& \leq C\left(\int_{0}^{\infty} e^{-2 t z} d z\right)^{1 / 2}\left\|h_{\lambda}(f)\right\|_{L^{2}(0, \infty)} \leq \frac{C}{t^{1 / 2}}\|f\|_{L^{2}(0, \infty)}<\infty, \quad y, t \in(0, \infty),
\end{aligned}
$$

which allows us to establish (2.5). In analogous way the differentiation under the integral sign in (2.6) can be justified.

Also, since (see [19, (5.3.5)]),

$$
\partial_{y}\left[(y z)^{-\nu} J_{\nu}(y z)\right]=-z(y z)^{-\nu} J_{\nu+1}(y z), \quad y, z \in(0, \infty)
$$

it follows that

$$
\begin{aligned}
D_{\lambda, y}\left[P_{t}^{\lambda}(x, y)\right] & =y^{\lambda} \partial_{y} \int_{0}^{\infty}(y z)^{-\lambda+1 / 2} J_{\lambda-1 / 2}(y z) z^{\lambda} e^{-t z} \sqrt{x z} J_{\lambda-1 / 2}(x z) d z \\
& =-\int_{0}^{\infty} \sqrt{y z} J_{\lambda+1 / 2}(y z) z e^{-t z} \sqrt{x z} J_{\lambda-1 / 2}(x z) d z \\
& =-h_{\lambda+1}\left(z e^{-t z} \sqrt{x z} J_{\lambda-1 / 2}(x z)\right)(y), \quad x, y, t \in(0, \infty)
\end{aligned}
$$

and, for $f \in L^{2}(0, \infty)$,

$$
D_{\lambda, y}\left[P_{t}^{\lambda}(f)(y)\right]=-h_{\lambda+1}\left(z e^{-t z} h_{\lambda}(f)(z)\right)(y), \quad y, t \in(0, \infty) .
$$

Differentiation under the integral sign can be justified as above.

On the other hand, since $h_{\lambda}(f) \in L^{2}(0, \infty)$, the dominated convergence theorem implies that

$$
\lim _{t \rightarrow 0^{+}} t z e^{-t z} h_{\lambda}(f)(z)=\lim _{t \rightarrow+\infty} t z e^{-t z} h_{\lambda}(f)(z)=\lim _{t \rightarrow+\infty} e^{-t z} h_{\lambda}(f)(z)=0, \quad \text { in } L^{2}(0, \infty),
$$

and

$$
\lim _{t \rightarrow 0^{+}} e^{-t z} h_{\lambda}(f)(z)=h_{\lambda}(f)(z), \quad \text { in } L^{2}(0, \infty) .
$$

Then, from (2.5), (2.6) and the $L^{2}$-boundedness of $h_{\lambda}$ we get that

$$
\lim _{t \rightarrow 0^{+}} t \partial_{t}\left(P_{t}^{\lambda}(f)(z)\right)=\lim _{t \rightarrow+\infty} t \partial_{t}\left(P_{t}^{\lambda}(f)(z)\right)=\lim _{t \rightarrow+\infty} P_{t}^{\lambda}(f)(z)=0, \quad \text { in } L^{2}(0, \infty),
$$


and

$$
\lim _{t \rightarrow 0^{+}} P_{t}^{\lambda}(f)(z)=f, \quad \text { in } L^{2}(0, \infty) .
$$

Our next objective is to prove the following lemma.

Lemma 2.2. Let $f \in L^{2}(0, \infty)$. Then,

$$
f(x)=2 \lim _{\varepsilon \rightarrow 0^{+}} \int_{\varepsilon}^{1 / \varepsilon} \int_{0}^{\infty} t \nabla_{\lambda, y}\left(P_{t}^{\lambda}(x, y)\right) \cdot \nabla_{\lambda, y}\left(P_{t}^{\lambda}(f)(y)\right) d y d t,
$$

where the equality is understood in $L^{2}(0, \infty)$ and also in a distributional sense.

Proof. By using (2.4) and (2.6), Plancherel inequality for $h_{\lambda}$ leads to

$$
\begin{aligned}
\int_{0}^{\infty} \partial_{t} P_{t}^{\lambda}(x, y) \partial_{t} P_{t}^{\lambda}(f)(y) d y & =\int_{0}^{\infty} h_{\lambda}\left(z e^{-t z} \sqrt{x z} J_{\lambda-1 / 2}(x z)\right)(y) h_{\lambda}\left(z e^{-t z} h_{\lambda}(f)(z)\right)(y) d y \\
& =\int_{0}^{\infty} z^{2} e^{-2 t z} \sqrt{x z} J_{\lambda-1 / 2}(x z) h_{\lambda}(f)(z) d z \\
& =\frac{1}{4} \partial_{t}^{2}\left[P_{2 t}^{\lambda}(f)(x)\right], \quad x, t \in(0, \infty) .
\end{aligned}
$$

In analogous way from (2.7), (2.8) and Plancherel equality for $h_{\lambda+1}$ we obtain

$$
\begin{aligned}
\int_{0}^{\infty} D_{\lambda, y} & {\left[P_{t}^{\lambda}(x, y)\right] D_{\lambda, y}\left[P_{t}^{\lambda}(f)(y)\right] d y } \\
& =\int_{0}^{\infty} h_{\lambda+1}\left(z e^{-t z} \sqrt{x z} J_{\lambda-1 / 2}(x z)\right)(y) h_{\lambda+1}\left(z e^{-t z} h_{\lambda}(f)(z)\right)(y) d y \\
& =\int_{0}^{\infty} z^{2} e^{-2 t z} h_{\lambda}(f)(z) \sqrt{x z} J_{\lambda-1 / 2}(x z) d z \\
& =\frac{1}{4} \partial_{t}^{2}\left[P_{2 t}^{\lambda}(f)(x)\right], \quad x, t \in(0, \infty) .
\end{aligned}
$$

By partial integration we obtain

$$
\begin{aligned}
\int_{\varepsilon}^{1 / \varepsilon} \int_{0}^{\infty} & t \nabla_{\lambda, y}\left(P_{t}^{\lambda}(x, y)\right) \cdot \nabla_{\lambda, y}\left(P_{t}^{\lambda}(f)(y)\right) d y d t=\frac{1}{2} \int_{\varepsilon}^{1 / \varepsilon} t \partial_{t}^{2}\left[P_{2 t}^{\lambda}(f)(x)\right] d t \\
= & \left.\left.\frac{1}{2}\left\{t \partial_{t}\left(P_{2 t}^{\lambda}(f)(x)\right)\right]_{t=\varepsilon}^{t=1 / \varepsilon}-P_{2 t}^{\lambda}(f)(x)\right]_{t=\varepsilon}^{t=1 / \varepsilon}\right\}, \quad x \in(0, \infty) \text { and } 0<\varepsilon<1 .
\end{aligned}
$$

We conclude from (2.9) and (2.10) that

$$
\lim _{\varepsilon \rightarrow 0^{+}} \int_{\varepsilon}^{1 / \varepsilon} \int_{0}^{\infty} t \nabla_{\lambda, y}\left(P_{t}^{\lambda}(x, y)\right) \cdot \nabla_{\lambda, y}\left(P_{t}^{\lambda}(f)(y)\right) d y d t=\frac{f(x)}{2}, \quad \text { in } L^{2}(0, \infty),
$$

and then, also, in a distributional sense.

\section{BMO SPACES ASSOCIATED With Bessel OPERATORS}

In this section we establish some properties for the functions in the space $B M O_{\mathrm{o}}(\mathbb{R})$ that will be useful in the proof of Theorem [1.1.

As it was mentioned in the introduction, in [5] Hardy spaces associated with Bessel operators $B_{\lambda}$ were introduced by using maximal operators. A function $f \in L^{1}(0, \infty)$ is in the Hardy space $H_{\lambda}^{1}(0, \infty)$ provided that the maximal function $P_{*}^{\lambda}(f) \in L^{1}(0, \infty)$. Here, $P_{*}^{\lambda}$ is defined by

$$
P_{*}^{\lambda}(f):=\sup _{t>0}\left|P_{t}^{\lambda}(f)\right|, \quad f \in L^{1}(0, \infty) .
$$

According to [5, Theorem 1.10 and Proposition 3.8] $H_{\lambda}^{1}(0, \infty)$ can be also defined by using the maximal operator associated to the heat semigroup $\left\{W_{t}^{\lambda}\right\}_{t>0}$ generated by $-B_{\lambda}$.

The area integral defined by the Poisson semigroup $\left\{P_{t}^{\lambda}\right\}_{t>0}, g_{\lambda}(f)$ of $f \in L^{1}(0, \infty)$ is defined by

$$
g_{\lambda}(f)(x):=\left(\int_{\Gamma_{+}(x)}\left|t \partial_{t} P_{t}^{\lambda}(f)(y)\right|^{2} \frac{d t d y}{t^{2}}\right)^{1 / 2}, \quad x \in(0, \infty),
$$

where $\Gamma_{+}(x):=\{(y, t) \in(0, \infty) \times(0, \infty):|x-y|<t\}, x \in(0, \infty)$. In [3. Proposition 4.1] it was proved that $f \in L^{1}(0, \infty)$ is in $H_{\lambda}^{1}(0, \infty)$ if and only if $g_{\lambda}(f) \in L^{1}(0, \infty)$. Actually, the space $H_{\lambda}^{1}(0, \infty)$ does not depend on $\lambda$ because, according to [5. Theorem 1.10] and [15. Theorem 2.1], a 
function $f \in L^{1}(0, \infty)$ is in $H_{\lambda}^{1}(0, \infty)$ when and only when the odd extension $f_{\mathrm{o}}$ of $f$ to $\mathbb{R}$ is in the classical Hardy space $H^{1}(\mathbb{R})$. Other characterizations for the space $H_{\lambda}^{1}(0, \infty)$ can be found in [4] (even in the multiparametric case).

The dual space of $H_{\lambda}^{1}(0, \infty)$ is the space $B M O_{\mathrm{o}}([3$, p. 466]). By using duality and the description of $H_{\lambda}^{1}(0, \infty)$ in terms of $g_{\lambda}$ we deduce a new characterization of $B M O_{\mathrm{o}}(\mathbb{R})$.

According to (2.3) we have that $P_{t}^{\lambda}(f)(x)<\infty$, for every $x, t \in(0, \infty)$, provided that $f$ is a complex measurable function on $(0, \infty)$ such that

$$
\int_{0}^{\infty} \frac{|f(x)|}{(1+x)^{2}} d x<\infty .
$$

We say that a function $f \in L^{1}\left((0, \infty),(1+x)^{-2} d x\right)$ is in $B M O\left(P^{\lambda}\right)$ when

$$
\|f\|_{B M O\left(P^{\lambda}\right)}:=\sup \frac{1}{|I|} \int_{I}\left|f(x)-P_{|I|}^{\lambda}(f)(x)\right| d x<\infty,
$$

where the supremum is taken over all bounded intervals $I$ in $(0, \infty)$.

We now characterize $B M O\left(P^{\lambda}\right)$ as the dual space of $H_{\lambda}^{1}(0, \infty)$. In order to do this we consider the odd-atoms introduced in [15]. A measurable function $\mathfrak{a}$ on $(0, \infty)$ is an odd-atom when it satisfies one of the following properties:

(a) $\mathfrak{a}=\frac{1}{\delta} \chi_{(0, \delta)}$, for some $\delta>0$. Here $\chi_{(0, \delta)}$ denotes the characteristic function of $(0, \delta)$, for every $\delta>0$.

(b) There exists a bounded interval $I \subset(0, \infty)$ such that $\operatorname{supp} \mathfrak{a} \subset I, \int_{I} \mathfrak{a}(x) d x=0$ and $\|\mathfrak{a}\|_{\infty} \leq|I|^{-1}$.

We say that a function $f \in L^{1}(0, \infty)$ is in $H_{\mathrm{o}, a t}^{1}(0, \infty)$ when, for every $j \in \mathbb{N}$, there exist $\lambda_{j}>0$ and an odd-atom $\mathfrak{a}_{j}$ such that $f=\sum_{j \in \mathbb{N}} \lambda_{j} \mathfrak{a}_{j}$, in $L^{1}(0, \infty)$, and $\sum_{j \in \mathbb{N}} \lambda_{j}<\infty$. We define, for every $f \in H_{\mathrm{o}, a t}^{1}(0, \infty)$,

$$
\|f\|_{H_{\mathrm{o}, a t}^{1}(0, \infty)}:=\inf \sum_{j \in \mathbb{N}} \lambda_{j}
$$

where the infimum is taken over all the sequences $\left\{\lambda_{j}\right\}_{j \in \mathbb{N}} \subset(0, \infty)$ such that $\sum_{j \in \mathbb{N}} \lambda_{j}<\infty$ and $f=\sum_{j \in \mathbb{N}} \lambda_{j} \mathfrak{a}_{j}$, in $L^{1}(0, \infty)$, where $\mathfrak{a}_{j}$ is an odd-atom, for every $j \in \mathbb{N}$.

According to [5, Proposition 3.7] we have that $H_{\mathrm{o}, a t}^{1}(0, \infty)=H_{\lambda}^{1}(0, \infty)$ algebraic and topologically. Note that this equality implies that $\mathcal{A}=\operatorname{span}\{$ odd atoms $\}$ is a dense subspace of $H_{\lambda}^{1}(0, \infty)$.

We now characterize $B M O\left(P^{\lambda}\right)$ as the dual space of $H_{\lambda}^{1}(0, \infty)$.

Proposition 3.1. Let $\lambda>0$.

i) Let $f \in B M O\left(P^{\lambda}\right)$. We define the functional $T_{f}$ on $\mathcal{A}$ by

$$
T_{f}(b):=\int_{0}^{\infty} f(x) b(x) d x, \quad b \in \mathcal{A} .
$$

Then, $T_{f}$ can be extended to $H_{\lambda}^{1}(0, \infty)$ as a bounded operator from $H_{\lambda}^{1}(0, \infty)$ into $\mathbb{C}$. Furthermore,

$$
\left\|T_{f}\right\|_{\left(H_{\lambda}^{1}(0, \infty)\right)^{\prime}} \leq C\|f\|_{B M O\left(P^{\lambda}\right)},
$$

where $C>0$ does not depend on $f$.

ii) There exists $C>0$ such that, for every $T \in\left(H_{\lambda}^{1}(0, \infty)\right)^{\prime}$, there exists $f \in B M O\left(P^{\lambda}\right)$ such that $T=T_{f}$ on $\mathcal{A}$ and

$$
\|f\|_{B M O\left(P^{\lambda}\right)} \leq C\|T\|_{\left(H_{\lambda}^{1}(0, \infty)\right)^{\prime}}
$$

Proof of Proposition [3.1, $i)$. Since $f \in B M O\left(P^{\lambda}\right)$, we can affirm that the measure $\rho_{f}$ on $(0, \infty) \times$ $(0, \infty)$ defined by

$$
d \rho_{f}(x, t):=\left|t\left(\partial_{t} P_{t}^{\lambda}\right)\left(i_{d}-P_{t}^{\lambda}\right) f(x)\right|^{2} \frac{d x d t}{t},
$$

where $i_{d}$ represents the identity operator, is Carleson and

$$
\left\|\rho_{f}\right\|_{\mathcal{C}} \leq C\|f\|_{B M O\left(P^{\lambda}\right)}^{2},
$$

with $C>0$. 
In order to prove this assertion, we can proceed as in the proof of [11, Lemma 4.6]. Indeed, it is sufficient to see that, there exists $C>0$ such that, for every bounded interval $I \subset(0, \infty)$ we have that

$$
\int_{\widehat{I}}\left|t\left(\partial_{t} P_{t}^{\lambda}\right)\left(i_{d}-P_{t}^{\lambda}\right)\left(i_{d}-P_{|I|}^{\lambda}\right) f(x)\right|^{2} \frac{d x d t}{t} \leq C|I|\|f\|_{B M O\left(P^{\lambda}\right)}^{2}
$$

and

$$
\int_{\widehat{I}}\left|t\left(\partial_{t} P_{t}^{\lambda}\right)\left(i_{d}-P_{t}^{\lambda}\right) P_{|I|}^{\lambda}(f)(x)\right|^{2} \frac{d x d t}{t} \leq C|I|\|f\|_{B M O\left(P^{\lambda}\right)}^{2} .
$$

We consider the Littlewood-Paley type function $G_{\lambda}$ defined by

$$
G_{\lambda}(g)(x):=\left(\int_{0}^{\infty}\left|t\left(\partial_{t} P_{t}^{\lambda}\right)\left(i_{d}-P_{t}^{\lambda}\right) g(x)\right|^{2} \frac{d t}{t}\right)^{1 / 2}, \quad x \in(0, \infty) .
$$

Let $g \in L^{2}(0, \infty)$. According to (2.5), (2.6) and the fact that $h_{\lambda}^{2}=i_{d}$, we can write

$$
t\left(\partial_{t} P_{t}^{\lambda}\right)\left(i_{d}-P_{t}^{\lambda}\right) g=-h_{\lambda}\left(t z e^{-t z}\left(1-e^{-t z}\right) h_{\lambda}(g)\right), \quad t>0 .
$$

Then, since $h_{\lambda}$ is a bounded operator from $L^{2}(0, \infty)$ into itself, we get

$$
\begin{aligned}
\left\|G_{\lambda}(g)\right\|_{2} & =\left(\int_{0}^{\infty} \int_{0}^{\infty}\left|h_{\lambda}\left(t z e^{-t z}\left(1-e^{-t z}\right) h_{\lambda}(g)\right)(x)\right|^{2} d x \frac{d t}{t}\right)^{1 / 2} \\
& \leq C\left(\int_{0}^{\infty} \int_{0}^{\infty}\left|t z e^{-t z}\left(1-e^{-t z}\right)\right|^{2} \frac{d t}{t}\left|h_{\lambda}(g)(z)\right|^{2} d z\right)^{1 / 2} \\
& \leq C\|g\|_{2} .
\end{aligned}
$$

Hence, the sublinear operator $G_{\lambda}$ is bounded from $L^{2}(0, \infty)$ into itself.

Let $I$ be a bounded interval in $(0, \infty)$.

We now decompose the function $\left(i_{d}-P_{|I|}^{\lambda}\right) f$ as follows:

$$
\left(i_{d}-P_{|I|}^{\lambda}\right) f=\chi_{2 I}\left(i_{d}-P_{|I|}^{\lambda}\right) f+\chi_{(0, \infty) \backslash 2 I}\left(i_{d}-P_{|I|}^{\lambda}\right) f=: g_{1}+g_{2} .
$$

The arguments in [11, p. 956] (see also [12]) allow us to obtain

$$
\int_{\widehat{I}}\left|t\left(\partial_{t} P_{t}^{\lambda}\right)\left(i_{d}-P_{t}^{\lambda}\right)\left(g_{1}\right)(x)\right|^{2} \frac{d x d t}{t} \leq\left\|G_{\lambda}\left(g_{1}\right)\right\|_{2}^{2} \leq C\left\|g_{1}\right\|_{2}^{2} \leq C|I|\|f\|_{B M O\left(P^{\lambda}\right)}^{2},
$$

and also, by using Lemma 2.1 and (2.3), that

$$
\left|t\left(\partial_{t} P_{t}^{\lambda}\right)\left(i_{d}-P_{t}^{\lambda}\right)\left(g_{2}\right)(x)\right| \leq C \frac{t}{|I|}\|f\|_{B M O\left(P^{\lambda}\right)}
$$

Then, we get

$$
\int_{\widehat{I}}\left|t\left(\partial_{t} P_{t}^{\lambda}\right)\left(i_{d}-P_{t}^{\lambda}\right)\left(g_{2}\right)(x)\right|^{2} \frac{d x d t}{t} \leq C|I|\|f\|_{B M O\left(P^{\lambda}\right)}^{2}
$$

Inequality (3.1) follows now from (3.3) and (3.4).

According again to Lemma 2.1, (2.3) and by proceeding as in the bottom of [11, p. 956] we obtain

$$
\left|t\left(\partial_{t} P_{t}^{\lambda}\right)\left(i_{d}-P_{t}^{\lambda}\right) P_{|I|}^{\lambda}(f)(x)\right| \leq C \frac{t}{|I|}\|f\|_{B M O\left(P^{\lambda}\right)},
$$

and 3.2 can be established.

If $F$ is a measurable function on $(0, \infty) \times(0, \infty)$ we define (see [3, p. 488])

$$
\Phi(F)(x):=\sup _{I \subset(0, \infty), I \text { bounded }, x \in I}\left(\frac{1}{|I|} \int_{0}^{|I|} \int_{I}|F(y, t)|^{2} \frac{d y d t}{t}\right)^{1 / 2}, \quad x \in(0, \infty),
$$

and

$$
\Psi(F)(x):=\left(\int_{\Gamma_{+}(x)}|F(y, t)|^{2} \frac{d y d t}{t^{2}}\right)^{1 / 2}, \quad x \in(0, \infty) .
$$

Suppose that $b \in \mathcal{A}$ and consider $F(x, t):=t\left(\partial_{t} P_{t}^{\lambda}\right)\left(i_{d}-P_{t}^{\lambda}\right) f$ and $G(x, t):=t \partial_{t} P_{t}^{\lambda}(b)(x)$, $x, t \in(0, \infty)$. Since $\rho_{f}$ is a Carleson measure we have that $\Phi(F) \in L^{\infty}(0, \infty)$ and

$$
\|\Phi(F)\|_{\infty} \leq C\left\|\rho_{f}\right\|_{\mathcal{C}}^{1 / 2}
$$


On the other hand, from [3. Proposition 4.1], we have that $\Psi(G) \in L^{1}(0, \infty)$ and $\|\Psi(G)\|_{1} \leq$ $C\|b\|_{H_{\lambda}^{1}(0, \infty)}$. Then, according to [3, Proposition 4.3] we get

$$
\begin{aligned}
\int_{0}^{\infty} & \int_{0}^{\infty}\left|t\left(\partial_{t} P_{t}^{\lambda}\right)\left(i_{d}-P_{t}^{\lambda}\right) f(y) \| t \partial_{t} P_{t}^{\lambda}(b)(y)\right| \frac{d y d t}{t} \\
& \leq C \int_{0}^{\infty} \Phi(F)(y) \Psi(G)(y) d y \leq C\|f\|_{B M O\left(P^{\lambda}\right)}\|b\|_{H_{\lambda}^{1}(0, \infty)} .
\end{aligned}
$$

It follows that

$$
\int_{0}^{\infty} \int_{0}^{\infty} t\left(\partial_{t} P_{t}^{\lambda}\right)\left(i_{d}-P_{t}^{\lambda}\right) f(y) t \partial_{t} P_{t}^{\lambda}(b)(y) \frac{d y d t}{t}=\lim _{\varepsilon \rightarrow 0^{+}, N \rightarrow \infty} H(\varepsilon, N),
$$

where, for every $0<\varepsilon<N<\infty$,

We can write

$$
H(\varepsilon, N):=\int_{\varepsilon}^{N} \int_{0}^{\infty} t\left(\partial_{t} P_{t}^{\lambda}\right)\left(i_{d}-P_{t}^{\lambda}\right) f(y) t \partial_{t} P_{t}^{\lambda}(b)(y) \frac{d y d t}{t} .
$$

$$
t\left(\partial_{t} P_{t}^{\lambda}\right)\left(i_{d}-P_{t}^{\lambda}\right)=t \partial_{t} P_{t}^{\lambda}-\frac{1}{2} t \partial_{t} P_{2 t}^{\lambda}, \quad t>0
$$

Since $f \in L^{1}\left((0, \infty),(1+x)^{-2} d x\right)$ and $b \in \mathcal{A}$, by using [3, (4.8)] we deduce that

$$
\begin{aligned}
& \int_{0}^{\infty} t\left(\partial_{t} P_{t}^{\lambda}\right)\left(i_{d}-P_{t}^{\lambda}\right) f(y) \partial_{t} P_{t}^{\lambda}(b)(y) d y \\
& \quad=\int_{0}^{\infty} f(z) \int_{0}^{\infty} t\left(\partial_{t} P_{t}^{\lambda}(y, z)-\frac{1}{2} \partial_{t} P_{2 t}^{\lambda}(y, z)\right) \partial_{t} P_{t}^{\lambda}(b)(y) d y d z, \quad t>0
\end{aligned}
$$

and, for every $0<\varepsilon<N<\infty$,

$$
H(\varepsilon, N)=\int_{0}^{\infty} f(z) \int_{\varepsilon}^{N} \int_{0}^{\infty} t\left(\partial_{t} P_{t}^{\lambda}(y, z)-\frac{1}{2} \partial_{t} P_{2 t}^{\lambda}(y, z)\right) \partial_{t} P_{t}^{\lambda}(b)(y) d y d t d z
$$

According to [3, (4.15)] we obtain

$$
\lim _{\varepsilon \rightarrow 0^{+}, N \rightarrow \infty} \int_{\varepsilon}^{N} \int_{0}^{\infty} t \partial_{t} P_{t}^{\lambda}(y, z) \partial_{t} P_{t}^{\lambda}(b)(y) d y d t=\frac{b(z)}{4}, \quad \text { in } L^{2}(0, \infty) .
$$

In a similar way we can see that

$$
\lim _{\varepsilon \rightarrow 0^{+}, N \rightarrow \infty} \int_{\varepsilon}^{N} \int_{0}^{\infty} t \partial_{t} P_{2 t}^{\lambda}(y, z) \partial_{t} P_{t}^{\lambda}(b)(y) d y d t=\frac{2 b(z)}{9}, \quad \text { in } L^{2}(0, \infty) .
$$

Then,

$$
\lim _{\varepsilon \rightarrow 0^{+}, N \rightarrow \infty} \int_{\varepsilon}^{N} \int_{0}^{\infty} t\left(\partial_{t} P_{t}^{\lambda}(y, z)-\frac{1}{2} \partial_{t} P_{2 t}^{\lambda}(y, z)\right) \partial_{t} P_{t}^{\lambda}(b)(y) d y d t=\frac{5 b(z)}{36}
$$

in $L^{2}(0, \infty)$. By using now dominated convergence theorem as in [3, p. 492] we conclude that

$$
\int_{0}^{\infty} \int_{0}^{\infty} t \partial_{t}\left(P_{t}^{\lambda}\right)\left(i_{d}-P_{t}^{\lambda}\right) f(x) \partial_{t} P_{t}^{\lambda}(b)(x) \frac{d x d t}{t}=\frac{5}{36} \int_{0}^{\infty} f(x) b(x) d x .
$$

By combining (3.5) and (3.6) we get

$$
\left|T_{f}(b)\right|=\left|\int_{0}^{\infty} f(x) b(x) d x\right| \leq C\|f\|_{B M O\left(P^{\lambda}\right)}\|b\|_{H_{\lambda}^{1}(0, \infty)} .
$$

Proof of Proposition 3.1, ii). Assume that $T \in\left(H_{\lambda}^{1}(0, \infty)\right)^{\prime}$. There exists $f \in B M O_{\mathrm{o}}(\mathbb{R})$ such that $T g=T_{f} g$, for every $g \in \mathcal{A}$, and $\|f\|_{B M O_{\mathrm{o}}(\mathbb{R})} \leq C\|T\|_{\left(H_{\lambda}^{1}(0, \infty)\right)^{\prime}}([\underline{3}$, p. 466] and [5, Theorem 1.10]). We are going to see that $f \in B M O\left(P^{\lambda}\right)$.

Let $I$ be a bounded interval in $(0, \infty)$. We can write

$$
\begin{aligned}
\frac{1}{|I|} \int_{I}\left|f(x)-P_{|I|}^{\lambda}(f)(x)\right| d x \leq & \frac{1}{|I|} \int_{I}\left|f(x)-f_{I}\right| d x+\frac{1}{|I|} \int_{I}\left|P_{|I|}^{\lambda}\left(f-f_{I}\right)(x)\right| d x \\
& +\frac{\left|f_{I}\right|}{|I|} \int_{I}\left|P_{|I|}^{\lambda}(1)(x)-1\right| d x=: J_{1}+J_{2}+J_{3} .
\end{aligned}
$$


Since $f \in B M O_{\mathrm{o}}(\mathbb{R}), J_{1} \leq\|f\|_{B M O_{\mathrm{o}}(\mathbb{R})}$. According to (2.3) we have that

$$
\begin{aligned}
\left|P_{|I|}^{\lambda}\left(f-f_{I}\right)(x)\right| & \leq C \int_{0}^{\infty} \frac{|I|}{|x-y|^{2}+|I|^{2}}\left|f(y)-f_{I}\right| d y \\
& \leq C\left(\int_{(0, \infty) \cap(x-|I| / 2, x+|I| / 2)}+\sum_{k \in \mathbb{N}} \int_{(0, \infty) \cap\left(B\left(x, 2^{k}|I|\right) \backslash B\left(x, 2^{k-1}|I|\right)\right.}\right) \frac{|I|\left|f(y)-f_{I}\right|}{(x-y)^{2}+|I|^{2}} d y \\
& \leq C\left(\frac{1}{|I|} \int_{(0, \infty) \cap(2 I)}\left|f(y)-f_{I}\right| d y+\sum_{k \in \mathbb{N}} \frac{1}{2^{2 k}|I|} \int_{(0, \infty) \cap 2^{k+2} I}\left|f(y)-f_{I}\right| d y\right) \\
& \leq C\|f\|_{B M O_{\circ}(\mathbb{R})}\left(1+\sum_{k \in \mathbb{N}} \frac{k}{2^{k}}\right) \leq C\|f\|_{B M O_{\circ}(\mathbb{R})}, \quad x \in I .
\end{aligned}
$$

Then, $J_{2} \leq C\|f\|_{B M O_{\mathrm{o}}(\mathbb{R})}$.

In order to estimate $J_{3}$ we distinguish two cases. We consider firstly that $x_{I} \leq|I|$. According to (2.3) we get

$$
\left|P_{|I|}^{\lambda}(1)(x)-1\right| \leq C \int_{0}^{\infty} \frac{|I|}{(x-y)^{2}+|I|^{2}} d y+1 \leq C, \quad x \in(0, \infty) .
$$

Then

$$
J_{3} \leq C\left|f_{I}\right| \leq \frac{C}{|I|} \int_{I}|f(y)| d y \leq C \frac{x_{I}+|I|}{|I|\left(x_{I}+|I|\right)} \int_{0}^{x_{I}+|I|}|f(y)| d y \leq C\|f\|_{B M O_{\circ}(\mathbb{R})} .
$$

Suppose now that $I=\left(x_{I}-|I| / 2, x_{I}+|I| / 2\right)$, where $x_{I}>|I|$. We write

$$
\begin{aligned}
P_{|I|}^{\lambda}(1)(x)-1= & \int_{0}^{\infty} P_{|I|}^{\lambda}(x, y) d y-\frac{1}{\pi} \int_{-\infty}^{+\infty} \frac{|I|}{(x-y)^{2}+|I|^{2}} d y \\
= & \left(\int_{0}^{x / 2}+\int_{2 x}^{\infty}\right) P_{|I|}^{\lambda}(x, y) d y-\frac{1}{\pi}\left(\int_{-\infty}^{x / 2}+\int_{2 x}^{\infty}\right) \frac{|I|}{(x-y)^{2}+|I|^{2}} d y \\
& +\int_{x / 2}^{2 x}\left(P_{|I|}^{\lambda}(x, y)-\frac{1}{\pi} \frac{|I|}{(x-y)^{2}+|I|^{2}}\right) d y \\
= & : \sum_{i=1}^{3} R_{i}(x), \quad x \in(0, \infty) .
\end{aligned}
$$

By using (2.3) we get

$$
\begin{aligned}
\left|R_{1}(x)+R_{2}(x)\right| & \leq C\left\{\left(\int_{0}^{x / 2}+\int_{2 x}^{\infty}\right) \frac{|I|}{(x-y)^{2}+|I|^{2}} d y+\int_{0}^{\infty} \frac{|I|}{(x+y)^{2}+|I|^{2}} d y\right\} \\
& \leq C|I|\left\{\left(\int_{0}^{x / 2}+\int_{2 x}^{\infty}\right) \frac{1}{(x-y)^{2}+|I|^{2}} d y+\int_{x / 2}^{2 x} \frac{1}{(x+y)^{2}+|I|^{2}} d y\right\} \\
& \leq C|I|\left(\int_{0}^{x / 2} \frac{d y}{x^{2}}+\int_{x / 2}^{\infty} \frac{d y}{y^{2}}\right) \leq C \frac{|I|}{x}, \quad x \in(0, \infty) .
\end{aligned}
$$

To analyze $R_{3}(x), x \in(0, \infty)$, we write

$$
\begin{aligned}
P_{t}^{\lambda}(x, y) & =\frac{2 \lambda t(x y)^{\lambda}}{\pi}\left(\int_{0}^{\pi / 2}+\int_{\pi / 2}^{\pi}\right) \frac{(\sin \theta)^{2 \lambda-1}}{\left((x-y)^{2}+t^{2}+2 x y(1-\cos \theta)\right)^{\lambda+1}} d \theta \\
& =: P_{t}^{\lambda, 1}(x, y)+P_{t}^{\lambda, 2}(x, y), \quad x, y, t \in(0, \infty),
\end{aligned}
$$

and

$$
\begin{aligned}
P_{t}^{\lambda, 1}(x, y)= & \frac{2 \lambda t(x y)^{\lambda}}{\pi}\left\{\int_{0}^{\pi / 2} \frac{(\sin \theta)^{2 \lambda-1}-\theta^{2 \lambda-1}}{\left((x-y)^{2}+t^{2}+2 x y(1-\cos \theta)\right)^{\lambda+1}} d \theta\right. \\
& +\int_{0}^{\pi / 2}\left(\frac{\theta^{2 \lambda-1}}{\left((x-y)^{2}+t^{2}+2 x y(1-\cos \theta)\right)^{\lambda+1}}-\frac{\theta^{2 \lambda-1}}{\left((x-y)^{2}+t^{2}+x y \theta^{2}\right)^{\lambda+1}}\right) d \theta \\
& \left.+\int_{0}^{\infty} \frac{\theta^{2 \lambda-1}}{\left((x-y)^{2}+t^{2}+x y \theta^{2}\right)^{\lambda+1}} d \theta-\int_{\pi / 2}^{\infty} \frac{\theta^{2 \lambda-1}}{\left((x-y)^{2}+t^{2}+x y \theta^{2}\right)^{\lambda+1}} d \theta\right\}
\end{aligned}
$$




$$
=: \frac{2 \lambda t(x y)^{\lambda}}{\pi} \sum_{i=1}^{4} I_{i}(x, y, t), \quad x, y, t \in(0, \infty) .
$$

We observe that

$$
\frac{2 \lambda t(x y)^{\lambda}}{\pi} I_{3}(x, y, t)=\frac{1}{\pi} \frac{t}{(x-y)^{2}+t^{2}}=P_{t}(x-y), \quad x, y, t \in(0, \infty),
$$

and then, we obtain, for each $x, y, t \in(0, \infty)$,

$$
P_{t}^{\lambda}(x, y)-P_{t}(x-y)=\frac{2 \lambda t(x y)^{\lambda}}{\pi}\left(I_{1}(x, y, t)+I_{2}(x, y, t)+I_{4}(x, y, t)\right)+P_{t}^{\lambda, 2}(x, y) .
$$

We have that,

$$
\left|P_{t}^{\lambda, 2}(x, y)\right| \leq C \frac{t(x y)^{\lambda}}{\left(x^{2}+y^{2}+t^{2}\right)^{\lambda+1}} \leq C \frac{t}{x^{2}}, \quad x, y, t \in(0, \infty) .
$$

By using mean value theorem we get

$$
\begin{aligned}
\left|I_{1}(x, y, t)\right| & \leq C \int_{0}^{\pi / 2} \frac{\theta^{2 \lambda+1}}{\left((x-y)^{2}+t^{2}+x y \theta^{2}\right)^{\lambda+1}} d \theta \leq C \int_{0}^{\pi / 2} \frac{\theta^{2 \lambda+1}}{(|x-y|+t+x \theta)^{2 \lambda+2}} d \theta \\
& \leq \frac{C}{x^{2 \lambda+3 / 2}|x-y|^{1 / 2}}, \quad 0<\frac{x}{2}<y<2 x, t>0
\end{aligned}
$$

and

$$
\begin{aligned}
\left|I_{2}(x, y, t)\right| & \leq C \int_{0}^{\pi / 2} \frac{x y \theta^{2 \lambda+3}}{\left((x-y)^{2}+t^{2}+x y \theta^{2}\right)^{\lambda+2}} d \theta \leq C \int_{0}^{\pi / 2} \frac{x^{2} \theta^{2 \lambda+3}}{(|x-y|+t+x \theta)^{2 \lambda+4}} d \theta \\
& \leq \frac{C}{x^{2 \lambda+3 / 2}|x-y|^{1 / 2}}, \quad 0<\frac{x}{2}<y<2 x, t>0 .
\end{aligned}
$$

Also, for every $t>0$ and $0<\frac{x}{2}<y<2 x$, we can write

$$
\begin{aligned}
\left|I_{4}(x, y, t)\right| & =\frac{(x y)^{-\lambda}}{(x-y)^{2}+t^{2}} \int_{\frac{\pi}{2} \sqrt{x y /\left((x-y)^{2}+t^{2}\right)}}^{\infty} \frac{u^{2 \lambda-1}}{\left(1+u^{2}\right)^{\lambda+1}} d u \\
& \leq C \frac{x^{-2 \lambda}}{(x-y)^{2}+t^{2}} \int_{\frac{\pi}{2} \sqrt{x y /\left((x-y)^{2}+t^{2}\right)}}^{\infty} \frac{d u}{u^{3}} \leq \frac{C}{x^{2 \lambda+2}} .
\end{aligned}
$$

From (3.10) and by putting together the above estimates we get

$$
\left|P_{t}^{\lambda}(x, y)-P_{t}(x-y)\right| \leq C t\left(\frac{1}{x^{3 / 2}|x-y|^{1 / 2}}+\frac{1}{x^{2}}\right), \quad 0<\frac{x}{2}<y<2 x, t>0 .
$$

It follows that

$$
\left|R_{3}(x)\right| \leq C|I| \int_{x / 2}^{2 x}\left(\frac{1}{x^{3 / 2}|x-y|^{1 / 2}}+\frac{1}{x^{2}}\right) d y \leq C \frac{|I|}{x}, \quad x \in(0, \infty) .
$$

We obtain that

$$
\left|P_{|I|}^{\lambda}(1)(x)-1\right| \leq C \frac{|I|}{x}, \quad x \in(0, \infty) .
$$

Since $x_{I}>|I|$, then $x_{I}-|I| / 2>2^{-1} x_{I}$, and we get

$$
\begin{aligned}
J_{3} & \leq C\left|f_{I}\right| \int_{I} \frac{1}{x} d x \leq \frac{C}{|I|} \int_{I}|f(y)| d y \frac{|I|}{x_{I}-|I| / 2} \\
& \leq C \frac{x_{I}+|I|}{\left(x_{I}-|I| / 2\right)\left(x_{I}+|I|\right)} \int_{0}^{x_{I}+|I|}|f(y)| d y \leq C\|f\|_{B M O_{\circ}(\mathbb{R})} .
\end{aligned}
$$

We conclude that

$$
\frac{1}{|I|} \int_{I}\left|f(x)-P_{|I|}^{\lambda}(f)(x)\right| d x \leq C\|f\|_{B M O_{\circ}(\mathbb{R})} .
$$

Thus we prove that $f \in B M O\left(P^{\lambda}\right)$ and that

$$
\|f\|_{B M O\left(P^{\lambda}\right)} \leq C\|f\|_{B M O_{\circ}(\mathbb{R})} \leq C\|T\|_{\left(H_{1}^{\lambda}(0, \infty)\right)^{\prime}} .
$$


From Proposition 3.1 and since $B M O_{\circ}(\mathbb{R})$ is the dual space of $H_{\lambda}^{1}(0, \infty)$ ([3, p. 466]), we can deduce the equality of $B M O\left(P^{\lambda}\right)$ and $B M O_{o}(\mathbb{R})$.

Corollary 3.2. Let $\lambda>0$. Then, $B M O\left(P^{\lambda}\right)=B M O_{o}(\mathbb{R})$ algebraic and topologically.

The following property will be very useful in the sequel.

Proposition 3.3. Let $\lambda>0$. There exists $C>0$ such that, for every $f \in B M O_{o}(\mathbb{R})$,

$$
\left|t \partial_{t} P_{t}^{\lambda}(f)(x)\right|+\left|t D_{\lambda, x} P_{t}^{\lambda}(f)(x)\right| \leq C\|f\|_{B M O_{o}(\mathbb{R})}, \quad x, t \in(0, \infty) .
$$

Proof. Let $f \in B M O_{\mathrm{o}}(\mathbb{R})$ and consider the odd extension $f_{\mathrm{o}}$ of $f$ to $\mathbb{R}$. We have that $f_{\mathrm{o}} \in B M O(\mathbb{R})$ and

$$
P_{t}\left(f_{\mathrm{o}}\right)(x)=\int_{0}^{\infty}\left(P_{t}(x-y)-P_{t}(x+y)\right) f(y) d y, \quad x, t \in(0, \infty) .
$$

Since $f_{\mathrm{o}} \in B M O(\mathbb{R})$ and $\left\|f_{\mathrm{o}}\right\|_{B M O(\mathbb{R})} \leq C\|f\|_{B M O_{\mathrm{o}}(\mathbb{R})}$, from [29, (2), p. 22] (see also [16]) we deduce that

$$
\left|t \partial_{t} P_{t}\left(f_{\mathrm{o}}\right)(x)\right|+\left|t \partial_{x} P_{t}\left(f_{\mathrm{o}}\right)(x)\right| \leq C\|f\|_{B M O_{\mathrm{o}}(\mathbb{R})}, \quad t, x \in(0, \infty) .
$$

Also, we can write

$$
\begin{aligned}
t \partial_{t} P_{t}^{\lambda}(f)(x)= & t \partial_{t} P_{t}\left(f_{\mathrm{o}}\right)(x)+\int_{0}^{\infty} t \partial_{t}\left(P_{t}^{\lambda}(x, y)-P_{t}(x-y)+P_{t}(x+y)\right) f(y) d y \\
= & t \partial_{t} P_{t}\left(f_{\mathrm{o}}\right)(x)+\left(\int_{0}^{x / 2}+\int_{2 x}^{\infty}\right) t \partial_{t} P_{t}^{\lambda}(x, y) f(y) d y \\
& +\left(\int_{0}^{x / 2}+\int_{2 x}^{\infty}\right) t \partial_{t}\left(P_{t}(x+y)-P_{t}(x-y)\right) f(y) d y \\
& +\int_{x / 2}^{2 x} t \partial_{t} P_{t}(x+y) f(y) d y+\int_{x / 2}^{2 x} t \partial_{t}\left(P_{t}^{\lambda}(x, y)-P_{t}(x-y)\right) f(y) d y \\
= & t \partial_{t} P_{t}\left(f_{\mathrm{o}}\right)(x)+\sum_{i=1}^{4} J_{i}(x, t), \quad x, t \in(0, \infty) .
\end{aligned}
$$

By using Lemma 2.1 and (2.2) we deduce that

$$
\begin{aligned}
\left|J_{1}(x, t)\right| & \leq C\left(\int_{0}^{x / 2}+\int_{2 x}^{\infty}\right) \frac{t(x y)^{\lambda}|f(y)|}{\left((x-y)^{2}+t^{2}\right)^{\lambda+1}} d y \\
& \leq C\left(\int_{0}^{x / 2} \frac{t x^{2 \lambda}|f(y)|}{\left(x^{2}+t^{2}\right)^{\lambda+1}} d y+\int_{2 x}^{\infty} \frac{t(x y)^{\lambda}|f(y)|}{\left(y^{2}+t^{2}\right)^{\lambda+1}} d y\right) \\
& \leq C\left(\frac{1}{x} \int_{0}^{x}|f(y)| d y+x^{\lambda} \int_{2 x}^{\infty} \frac{|f(y)|}{y^{\lambda+1}} d y\right) \\
& \leq C\left(\|f\|_{B M O_{\circ}(\mathbb{R})}+x^{\lambda} \sum_{k=1}^{\infty} \int_{2^{k} x}^{2^{k+1} x} \frac{|f(y)|}{y^{\lambda+1}} d y\right) \\
& \leq C\left(\|f\|_{B M O_{\circ}(\mathbb{R})}+x^{\lambda} \sum_{k=1}^{\infty} \frac{1}{\left(2^{k} x\right)^{\lambda+1}} \int_{0}^{2^{k+1} x}|f(y)| d y\right) \\
& \leq C\left(\|f\|_{B M O_{\circ}(\mathbb{R})}+\sum_{k=1}^{\infty} 2^{-k \lambda} \frac{1}{2^{k+1} x} \int_{0}^{2^{k+1} x}|f(y)| d y\right) \\
& \leq C\|f\|_{B M O_{\circ}(\mathbb{R})}, \quad x, t \in(0, \infty) .
\end{aligned}
$$

On the other hand,

$$
\begin{aligned}
\left|J_{2}(x, t)\right|= & \frac{1}{\pi}\left|\left(\int_{0}^{x / 2}+\int_{2 x}^{\infty}\right) t \partial_{t}\left(\frac{4 x y t}{\left((x-y)^{2}+t^{2}\right)\left((x+y)^{2}+t^{2}\right)}\right) f(y) d y\right| \\
=\frac{1}{\pi} \mid\left(\int_{0}^{x / 2}+\int_{2 x}^{\infty}\right) & \frac{4 x y t}{\left((x-y)^{2}+t^{2}\right)\left((x+y)^{2}+t^{2}\right)} \\
& \quad-\frac{8 x y t^{3}\left((x+y)^{2}+(x-y)^{2}+2 t^{2}\right)}{\left((x-y)^{2}+t^{2}\right)^{2}\left((x+y)^{2}+t^{2}\right)^{2}} f(y) d y \mid
\end{aligned}
$$




$$
\begin{aligned}
& \leq C\left(\int_{0}^{x / 2}+\int_{2 x}^{\infty}\right) \frac{x y t}{\left((x-y)^{2}+t^{2}\right)\left((x+y)^{2}+t^{2}\right)}|f(y)| d y \\
& \leq C\left(\frac{t x^{2}}{\left(x^{2}+t^{2}\right)^{2}} \int_{0}^{x}|f(y)| d y+t x \int_{2 x}^{\infty} \frac{y|f(y)|}{\left(y^{2}+t^{2}\right)^{2}} d y\right) \\
& \leq C\left(\frac{1}{x} \int_{0}^{x}|f(y)| d y+x \int_{2 x}^{\infty} \frac{|f(y)|}{y^{2}} d y\right) \leq C\|f\|_{B M O_{\circ}(\mathbb{R})}, \quad x, t \in(0, \infty) .
\end{aligned}
$$

The last inequality is obtained by proceeding as in (3.14) for $\lambda=1$.

Also, we have that

$\left|J_{3}(x, t)\right|=\frac{1}{\pi}\left|\int_{x / 2}^{2 x}\left(\frac{t}{(x+y)^{2}+t^{2}}-\frac{2 t^{3}}{\left((x+y)^{2}+t^{2}\right)^{2}}\right) f(y) d y\right| \leq \frac{C}{x} \int_{x / 2}^{2 x}|f(y)| d y \leq C\|f\|_{B M O_{\mathrm{o}}(\mathbb{R})}$.

Finally, in order to estimate $J_{4}(x, t), x, t \in(0, \infty)$, we consider (3.10) and write, for each $x, y, t \in(0, \infty)$,

$$
t \partial_{t}\left(P_{t}^{\lambda}(x, y)-P_{t}(x-y)\right)=\frac{2 \lambda}{\pi}(x y)^{\lambda} \sum_{i=1,2,4} t \partial_{t}\left(t I_{i}(x, y, t)\right)+t \partial_{t} P_{t}^{\lambda, 2}(x, y) .
$$

By using the mean value theorem we get

$$
\begin{aligned}
\left|t \partial_{t}\left(t I_{1}(x, y, t)\right)\right| & =\left|t I_{1}(x, y, t)-2(\lambda+1) t^{3} \int_{0}^{\pi / 2} \frac{(\sin \theta)^{2 \lambda-1}-\theta^{2 \lambda-1}}{\left((x-y)^{2}+t^{2}+2 x y(1-\cos \theta)\right)^{\lambda+2}} d \theta\right| \\
& \leq C t \int_{0}^{\pi / 2} \frac{\left|(\sin \theta)^{2 \lambda-1}-\theta^{2 \lambda-1}\right|}{\left((x-y)^{2}+t^{2}+2 x y(1-\cos \theta)\right)^{\lambda+1}} d \theta \\
& \leq C t \int_{0}^{\pi / 2} \frac{\theta^{2 \lambda+1}}{(|x-y|+t+x \theta)^{2 \lambda+2}} d \theta \leq \frac{C}{x^{2 \lambda+1}}, \quad 0<\frac{x}{2}<y<2 x, t>0,
\end{aligned}
$$

and

$$
\begin{aligned}
\left|t \partial_{t}\left(t I_{2}(x, y, t)\right)\right| & =\mid t I_{2}(x, y, t)-2(\lambda+1) t^{3} \int_{0}^{\pi / 2}\left[\frac{\theta^{2 \lambda-1}}{\left((x-y)^{2}+t^{2}+2 x y(1-\cos \theta)\right)^{\lambda+2}}\right. \\
& \left.-\frac{\theta^{2 \lambda-1}}{\left((x-y)^{2}+t^{2}+x y \theta^{2}\right)^{\lambda+2}}\right] d \theta \mid \\
& \leq C x y\left(\int_{0}^{\pi / 2} \frac{t \theta^{2 \lambda+3}}{\left((x-y)^{2}+t^{2}+x y \theta^{2}\right)^{\lambda+2}} d \theta+\int_{0}^{\pi / 2} \frac{t^{3} \theta^{2 \lambda+3}}{\left((x-y)^{2}+t^{2}+x y \theta^{2}\right)^{\lambda+3}} d \theta\right) \\
& \leq C t x^{2} \int_{0}^{\pi / 2} \frac{\theta^{2 \lambda+3}}{(|x-y|+t+x \theta)^{2 \lambda+4}} d \theta \leq \frac{C}{x^{2 \lambda+1}}, \quad 0<\frac{x}{2}<y<2 x, t>0 .
\end{aligned}
$$

Also, we obtain, when $0<x / 2<y<2 x$ and $t>0$,

$$
\begin{aligned}
\left|t \partial_{t}\left(t I_{4}(x, y, t)\right)\right| & =\left|t I_{4}(x, y, t)-2(\lambda+1) t^{3} \int_{\pi / 2}^{\infty} \frac{\theta^{2 \lambda-1}}{\left((x-y)^{2}+t^{2}+x y \theta^{2}\right)^{\lambda+2}} d \theta\right| \\
& \leq C t \int_{\pi / 2}^{\infty} \frac{\theta^{2 \lambda-1}}{\left((x-y)^{2}+t^{2}+x y \theta^{2}\right)^{\lambda+1}} d \theta \leq \frac{C}{x^{2 \lambda+1}} \int_{\pi / 2}^{\infty} \frac{d \theta}{\theta^{2}} \leq \frac{C}{x^{2 \lambda+1}} .
\end{aligned}
$$

Finally, we can write

$$
\begin{aligned}
\left|t \partial_{t}\left(P_{t}^{\lambda, 2}(x, y)\right)\right| & =\left|P_{t}^{\lambda, 2}(x, y)-\frac{4 \lambda(\lambda+1)}{\pi} t^{3}(x y)^{\lambda} \int_{\pi / 2}^{\pi} \frac{(\sin \theta)^{2 \lambda-1}}{\left((x-y)^{2}+t^{2}+2 x y(1-\cos \theta)\right)^{\lambda+2}} d \theta\right| \\
& \leq C t x^{2 \lambda} \int_{\pi / 2}^{\pi} \frac{\theta^{2 \lambda-1}}{(x+y+t)^{2 \lambda+2}} d \theta \leq \frac{C}{x}, \quad 0<\frac{x}{2}<y<2 x, t>0 . .
\end{aligned}
$$

From (3.15) and by combining the above estimates, it follows that

$$
J_{4}(x, t) \leq \frac{C}{x} \int_{0}^{2 x}|f(y)| d y \leq C\|f\|_{B M O_{\circ}(\mathbb{R})}, \quad x, t \in(0, \infty) .
$$

Equality (3.13) allows us to conclude that

$$
\left|t \partial_{t} P_{t}^{\lambda}(f)(x)\right| \leq C\|f\|_{B M O_{\circ}(\mathbb{R})}, \quad x, t \in(0, \infty) .
$$


We are going to see now that

$$
\left|t D_{\lambda, x} P_{t}^{\lambda}(f)(x)\right| \leq C\|f\|_{B M O_{\circ}(\mathbb{R})}, \quad x, t \in(0, \infty) .
$$

We use a decomposition similar to (3.13) and write

$$
\begin{aligned}
t D_{\lambda, x} P_{t}^{\lambda}(f)(x)= & t \partial_{x} P_{t}\left(f_{\mathrm{o}}\right)(x)+t \int_{0}^{\infty}\left[D_{\lambda, x}\left(P_{t}^{\lambda}(x, y)\right)-\partial_{x}\left(P_{t}(x-y)+P_{t}(x+y)\right)\right] f(y) d y \\
= & t \partial_{x} P_{t}\left(f_{\mathrm{o}}\right)(x)+\left(\int_{0}^{x / 2}+\int_{2 x}^{\infty}\right) t D_{\lambda, x}\left(P_{t}^{\lambda}(x, y)\right) f(y) d y \\
& +\left(\int_{0}^{x / 2}+\int_{2 x}^{\infty}\right) t \partial_{x}\left(P_{t}(x+y)-P_{t}(x-y)\right) f(y) d y \\
& +\int_{x / 2}^{2 x} t \partial_{x} P_{t}(x+y) f(y) d y+\int_{x / 2}^{2 x} t\left[D_{\lambda, x}\left(P_{t}^{\lambda}(x, y)\right)-\partial_{x}\left(P_{t}(x-y)\right)\right] f(y) d y \\
= & : t \partial_{x} P_{t}\left(f_{\mathrm{o}}\right)(x)+\sum_{i=1}^{4} H_{i}(x, t), \quad x, t \in(0, \infty) .
\end{aligned}
$$

By using Lemma 2.1 and (2.2) in the same way as in (3.14) we obtain

$$
\left|H_{1}(x, t)\right| \leq C\|f\|_{B M O_{\circ}(\mathbb{R})}, \quad x, t \in(0, \infty) .
$$

On the other hand,

$$
\begin{aligned}
& \left|H_{2}(x, t)\right|=\frac{1}{\pi}\left|\left(\int_{0}^{x / 2}+\int_{2 x}^{\infty}\right) \partial_{x}\left(\frac{4 x y t^{2}}{\left((x-y)^{2}+t^{2}\right)\left((x+y)^{2}+t^{2}\right)}\right) f(y) d y\right| \\
& \leq \frac{1}{\pi} \mid\left(\int_{0}^{x / 2}+\int_{2 x}^{\infty}\right) \frac{4 y t^{2}}{\left((x-y)^{2}+t^{2}\right)\left((x+y)^{2}+t^{2}\right)} \\
& \quad-4 x y t^{2} \frac{2(x-y)\left((x+y)^{2}+t^{2}\right)+2(x+y)\left((x-y)^{2}+t^{2}\right)}{\left((x-y)^{2}+t^{2}\right)^{2}\left((x+y)^{2}+t^{2}\right)^{2}} f(y) d y \mid \\
& \leq C\left(\int_{0}^{x / 2}+\int_{2 x}^{\infty}\right) \frac{y t^{2}}{\left((x-y)^{2}+t^{2}\right)\left((x+y)^{2}+t^{2}\right)}\left(1+\frac{x|x|}{(|x-y|+t)^{2}}+\frac{x(x+y)}{(x+y+t)^{2}}\right)|f(y)| d y \\
& \leq C\left(\frac{x t^{2}}{\left(x^{2}+t^{2}\right)^{2}} \int_{0}^{x}|f(y)| d y+\int_{2 x}^{\infty} \frac{y(y) \mid}{\left(y^{2}+t^{2}\right)(x+y+t)^{2}} d y\right) \\
& \leq C\left(\frac{1}{x} \int_{0}^{x}|f(y)| d y+\int_{2 x}^{\infty} \frac{t|f(y)|}{(x+y+t)^{2}} d y\right) \\
& \leq C\left(\|f\|_{B M O_{\circ}(\mathbb{R})}+\left(\int_{2 x}^{2 x+t}+\sum_{k=0}^{\infty} \int_{2 x+2^{k} t}^{2 x+2^{k+1} t}\right) \frac{t}{(x+y+t)^{2}}|f(y)| d y\right) \\
& \leq C\left(\|f\|_{B M O_{\circ}(\mathbb{R})}+\frac{1}{2 x+t} \int_{0}^{2 x+t}|f(y)| d y+\sum_{k=0}^{\infty} 2^{-k} \frac{1}{2 x+2^{k+1} t} \int_{0}^{2 x+2^{k+1} t}|f(y)| d y\right) \\
& \leq C\|f\|_{B M O_{\circ}(\mathbb{R}), \quad x, t \in(0, \infty) .}
\end{aligned}
$$

Also, it follows that

$$
\left|H_{3}(x, t)\right|=\frac{2}{\pi}\left|\int_{x / 2}^{2 x} \frac{(x+y) t^{2}}{\left((x+y)^{2}+t^{2}\right)^{2}} f(y) d y\right| \leq \frac{C}{x} \int_{0}^{2 x}|f(y)| d y \leq C\|f\|_{B M O_{\circ}(\mathbb{R})} .
$$

We deal now with $H_{4}(x, t), x, t \in(0, \infty)$. From (3.10) we have that

$$
\begin{aligned}
& D_{\lambda, x} P_{t}^{\lambda}(x, y)-\partial_{x} P_{t}(x-y)=\partial_{x}\left[P_{t}^{\lambda}(x, y)-P_{t}(x-y)\right]-\frac{\lambda}{x} P_{t}^{\lambda}(x, y) \\
& \quad=\frac{2 \lambda}{\pi} \sum_{i=1,2,4} t \partial_{x}\left[(x y)^{\lambda} I_{i}(x, y, t)\right]+\partial_{x} P_{t}^{\lambda, 2}(x, y)-\frac{\lambda}{x} P_{t}^{\lambda}(x, y), \quad x, y, t \in(0, \infty) .
\end{aligned}
$$

Again by using the mean value theorem we get

$$
\begin{aligned}
\left|t \partial_{x}\left[(x y)^{\lambda} I_{1}(x, y, t)\right]\right|= & \mid \frac{\lambda t}{x}(x y)^{\lambda} I_{1}(x, y, t) \\
& -2(\lambda+1) t(x y)^{\lambda} \int_{0}^{\pi / 2} \frac{\left[(\sin \theta)^{2 \lambda-1}-\theta^{2 \lambda-1}\right][(x-y)+y(1-\cos \theta)]}{\left((x-y)^{2}+t^{2}+2 x y(1-\cos \theta)\right)^{\lambda+2}} d \theta \mid
\end{aligned}
$$




$$
\begin{aligned}
& \leq C t x^{2 \lambda}\left(\frac{1}{x} \int_{0}^{\pi / 2} \frac{\theta^{2 \lambda+1}}{(|x-y|+t+x \theta)^{2 \lambda+2}} d \theta+\int_{0}^{\pi / 2} \frac{\theta^{2 \lambda+1}\left(|x-y|+y \theta^{2}\right)}{(|x-y|+t+x \theta)^{2 \lambda+4}} d \theta\right) \\
& \leq \frac{C}{t x}\left(\int_{0}^{\pi / 2} \theta d \theta+\int_{0}^{\pi / 2}(1+\theta) d \theta\right) \leq \frac{C}{t x}, \quad 0<\frac{x}{2}<y<2 x, t>0,
\end{aligned}
$$

and

$$
\begin{aligned}
\left|t \partial_{x}\left[(x y)^{\lambda} I_{2}(x, y, t)\right]\right|= & \mid \frac{\lambda t}{x}(x y)^{\lambda} I_{2}(x, y, t) \\
& -2(\lambda+1) t(x y)^{\lambda} \int_{0}^{\pi / 2} \theta^{2 \lambda-1}\left[\frac{(x-y)+y(1-\cos \theta)}{\left((x-y)^{2}+t^{2}+2 x y(1-\cos \theta)\right)^{\lambda+2}}\right. \\
\leq & C t(x y)^{\lambda}\left\{\frac{1}{x} \int_{0}^{\pi / 2} \frac{\theta^{2 \lambda+3} x y}{\left((x-y)^{2}+t^{2}+x y \theta^{2}\right)^{\lambda+2}}\right] d \theta \mid \\
& \left.+\int_{0}^{\pi / 2} \theta^{2 \lambda+3}\left(\frac{y \mid+t+x \theta)^{2 \lambda+4}}{(|x-y|+t+x \theta)^{2 \lambda+4}}+\frac{x y\left(|x-y|+y \theta^{2}\right)}{(|x-y|+t+x \theta)^{2 \lambda+6}}\right) d \theta\right\} \\
\leq & C\left(t x^{2 \lambda+1} \int_{0}^{\pi / 2} \frac{\theta^{2 \lambda+2}}{(|x-y|+t+x \theta)^{2 \lambda+4}} d \theta\right) \leq \frac{C}{t x}, \quad 0<\frac{x}{2}<y<2 x, t>0 .
\end{aligned}
$$

Now, we write

$$
\begin{aligned}
\left|t \partial_{x}\left((x y)^{\lambda} I_{4}(x, y, t)\right)\right|= & \mid \frac{\lambda t}{x}(x y)^{\lambda} I_{4}(x, y, t) \\
& -2(\lambda+1) t(x y)^{\lambda} \int_{\pi / 2}^{\infty} \frac{\theta^{2 \lambda-1}\left(x-y+y \theta^{2}\right)}{\left((x-y)^{2}+t^{2}+x y \theta^{2}\right)^{\lambda+2}} d \theta \mid \\
\leq & C t x^{2 \lambda-1} \int_{\pi / 2}^{\infty} \frac{\theta^{2 \lambda-1}}{(|x-y|+t+x \theta)^{2 \lambda+2}} d \theta \\
= & C \frac{t}{x(|x-y|+t)^{2}} \int_{\frac{\pi x}{2(|x-y|+t)}}^{\infty} \frac{u^{2 \lambda-1}}{(1+u)^{2 \lambda+2}} d u \leq \frac{C}{t x}, \quad 0<\frac{x}{2}<y<2 x, t>0 .
\end{aligned}
$$

Finally, it is clear from (2.3) that

and, also,

$$
\frac{1}{x}\left|P_{t}^{\lambda}(x, y)\right| \leq \frac{C}{t x}, \quad x, t \in(0, \infty),
$$

$$
\begin{aligned}
\left|\partial_{x}\left(P_{t}^{\lambda, 2}(x, y)\right)\right| & =\left|\frac{\lambda}{x} P_{t}^{\lambda, 2}(x, y)-\frac{4 \lambda(\lambda+1)}{\pi} t(x y)^{\lambda} \int_{\pi / 2}^{\pi} \frac{(\sin \theta)^{2 \lambda-1}[x-y+y(1-\cos \theta)]}{\left((x-y)^{2}+t^{2}+2 x y(1-\cos \theta)\right)^{\lambda+2}} d \theta\right| \\
& \leq C t x^{2 \lambda-1} \int_{\pi / 2}^{\pi} \frac{\theta^{2 \lambda-1}}{(x+y+t)^{2 \lambda+2}} d \theta \leq \frac{C}{t x}, \quad 0<\frac{x}{2}<y<2 x, t>0 .
\end{aligned}
$$

By combining the above estimates and taking into account (3.17) it follows that

$$
H_{4}(x, t) \leq \frac{C}{x} \int_{0}^{2 x}|f(y)| d y \leq C\|f\|_{B M O_{\circ}(\mathbb{R})}, \quad x, t \in(0, \infty) .
$$

Then, from (3.16) we conclude that

$$
\left|D_{\lambda, x} P_{t}^{\lambda}(f)(x)\right| \leq C\|f\|_{B M O_{o}(\mathbb{R})}, \quad x, t \in(0, \infty) .
$$

\section{Proof of Theorem 1.1}

Proof of Theorem 1.1, (i). Suppose that $\mu$ is a Carleson measure on $(0, \infty) \times(0, \infty)$. According to Corollary 3.2 in order to see that $S_{\mu, P^{\lambda}} \in B M O_{\mathrm{o}}(\mathbb{R})$ it is sufficient to see that there exists $C>0$ such that, for every bounded interval $I \subset(0, \infty)$,

$$
\frac{1}{|I|} \int_{I}\left|S_{\mu, P^{\lambda}}(x)-P_{|I|}^{\lambda}\left(S_{\mu, P^{\lambda}}\right)(x)\right| d x \leq C\|\mu\|_{\mathcal{C}}
$$


We proceed as in the proof of [8, Proposition 2.5].

Let $I$ be a bounded interval in $(0, \infty)$. We can write

$$
\begin{aligned}
\int_{I} \mid & S_{\mu, P^{\lambda}}(x)-P_{|I|}^{\lambda}\left(S_{\mu, P^{\lambda}}\right)(x) \mid d x \\
& \leq C \int_{I} \int_{(0, \infty)^{2}}\left|P_{t}^{\lambda}(x, y)-P_{t+|I|}^{\lambda}(x, y)\right| d \mu(y, t) d x \\
& \leq\left(\int_{I} \int_{\widehat{2 I}}+\int_{I} \int_{(0, \infty)^{2} \backslash \widehat{2 I}}\right)\left|P_{t}^{\lambda}(x, y)-P_{t+|I|}^{\lambda}(x, y)\right| d \mu(y, t) d x \\
& =: I_{1}+I_{2} .
\end{aligned}
$$

According to (2.3), since $\mu$ is a Carleson measure on $(0, \infty) \times(0, \infty)$, we get

$$
I_{1} \leq C \int_{\widehat{2 I}} \int_{I}\left(\frac{t}{(x-y)^{2}+t^{2}}+\frac{t+|I|}{(x-y)^{2}+(t+|I|)^{2}}\right) d x d \mu(y, t) \leq C \mu(\widehat{2 I}) \leq C|I|\|\mu\|_{\mathcal{C}} .
$$

Also by Lemmma 2.1 and (2.3), we obtain

$$
\begin{aligned}
I_{2} & \leq C \int_{I} \int_{(0, \infty)^{2} \backslash \widehat{2 I}} \int_{0}^{|I|}\left|\partial_{s} P_{t+s}^{\lambda}(x, y)\right| d s d \mu(y, t) d x \\
& \leq C \sum_{k=1}^{\infty} \int_{I} \int_{0}^{|I|} \int_{\widehat{2^{k+1} I} \backslash \widehat{2^{k} I}} \frac{1}{(x-y)^{2}+(s+t)^{2}} d \mu(y, t) d s d x \\
& \leq C \sum_{k=1}^{\infty} \mu\left(\widehat{2^{k+1} I}\right) \frac{1}{\left(2^{k+1}|I|\right)^{2}}|I|^{2} \leq C|I|\|\mu\|_{\mathcal{C}} .
\end{aligned}
$$

Thus, 4.1) is proved.

Proof of Theorem 1.1, (ii). We will use the procedure developed by Wilson ([29]) (see also [8]). We need to make modifications and to justify each step in our setting.

Let $Q$ be a bounded interval in $(0, \infty)$. In what follows we consider right-open intervals and denote by $x_{Q}$ the center of $Q$, and by $t_{Q}$ the length of $Q$.

Assume that $f \in B M O_{\mathrm{o}}(\mathbb{R})$ with supp $f \subset(0,1)$. We consider $u(x, t):=P_{t}^{\lambda}(f)(x), x, t \in$ $(0, \infty)$, and take $Q_{0}:=[0,2)$. In what follows we consider right-open intervals.

We now construct the $k$-th generation of subintervals of $Q_{0}$ as follows. By $A$ we denote a positive constant that will be fixed later. The 0 -th generation is defined by $G_{0}:=\left\{Q_{0}\right\}$. For every $k \in \mathbb{N}$, the $(k+1)$-th generation $G_{k+1}$ is defined recursively as follows. A dyadic interval $Q \subset Q_{0}$ is in $G_{k+1}$ when

(a) there exists $Q_{1} \in G_{k}$ such that $Q \subset Q_{1}$,

(b) $Q$ is a maximal dyadic with respect to the property

$$
\left|x_{Q}^{-\lambda} u\left(x_{Q}, t_{Q}\right)-x_{Q_{1}}^{-\lambda} u\left(x_{Q_{1}}, t_{Q_{1}}\right)\right|>A x_{Q}^{-\lambda} .
$$

Note that the properties of the dyadic intervals and the maximal property $(b)$ imply that, if $k \in \mathbb{N}$ and $Q_{1}, Q_{2} \in G_{k}$, then $Q_{1}=Q_{2}$ or $Q_{1} \cap Q_{2}=\emptyset$.

For every $k \in \mathbb{N}$ and $Q \in G_{k}$ we define the set

$$
\Sigma_{Q}:=\widehat{Q} \backslash \bigcup_{Q^{\prime} \subset Q, Q^{\prime} \in G_{k+1}} \widehat{Q^{\prime}}
$$

In the following figure where a possible $\Sigma_{Q}$ is represented, the dark grey squares are the Carleson boxes of those cubes $Q^{\prime} \subset Q$ that belong to $G_{k+1}$.

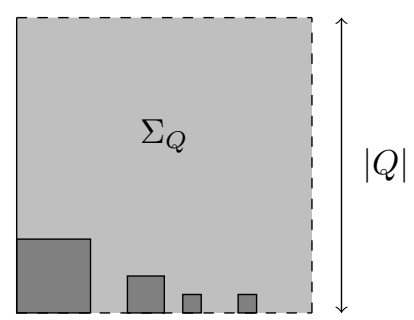

$Q$ 
The set $\Sigma_{Q}, Q \in G_{k}, k \in \mathbb{N}$, can be written in a different and useful way. For every interval $J$ we define $T(J)$ as follows

$$
T(J):=\left\{(x, t): x \in J \text { and } \frac{\ell(J)}{2} \leq t<\ell(J)\right\} .
$$

It is clear that, for every dyadic interval $S \subset(0, \infty)$, we have that

$$
\widehat{S}=\bigcup_{J \subset S, J \text { dyadic }} T(J) .
$$

Then, for every $k \in \mathbb{N}$ and $Q \in G_{k}$, we have that

$$
\Sigma_{Q}=\bigcup_{J \in \mathcal{A}(Q)} T(J)
$$

where $\mathcal{A}(Q):=\left\{J\right.$ dyadic $: J \subseteq Q, J \cap S^{\mathrm{c}} \neq \emptyset$, for every $\left.S \in G_{k+1}\right\}$.

Now, take $k \in \mathbb{N}$ and $Q \in G_{k}$. We are going to see that

$$
\left|x^{-\lambda} u(x, t)-x_{Q}^{-\lambda} u\left(x_{Q}, t_{Q}\right)\right| \leq C x^{-\lambda}\left(A+\|f\|_{B M O_{\circ}(\mathbb{R})}\right), \quad(x, t) \in \Sigma_{Q} .
$$

Here $C>0$ does not depend on $k$ or $Q$.

Suppose that $(x, t) \in \Sigma_{Q}$. There exists $J \in \mathcal{A}(Q)$ such that $(x, t) \in T(J)$. According to the definition of $G_{k+1}$ and since $x \leq 2 x_{J}$ we get

$$
\left|x_{Q}^{-\lambda} u\left(x_{Q}, t_{Q}\right)-x_{J}^{-\lambda} u\left(x_{J}, t_{J}\right)\right| \leq A x_{J}^{-\lambda} \leq 2^{\lambda} A x^{-\lambda} .
$$

On the other hand, for some $z$ in the segment joining $x$ and $x_{J}$ and for some $s$ in the segment joining $t$ and $t_{J}$, we have that

$$
x^{-\lambda} u(x, t)-x_{J}^{-\lambda} u\left(x_{J}, t_{J}\right)=\partial_{z}\left(z^{-\lambda} u(z, t)\right)\left(x_{J}-x\right)+\partial_{s}\left(x_{J}^{-\lambda} u\left(x_{J}, s\right)\right)\left(t_{J}-t\right) .
$$

Since $x \leq 2 x_{J}$, from Proposition 3.3 it follows that

$$
\left|x^{-\lambda} u(x, t)-x_{J}^{-\lambda} u\left(x_{J}, t_{J}\right)\right| \leq C x^{-\lambda}\|f\|_{B M O_{\circ}(\mathbb{R})},
$$

and (4.2) is checked.

Next, we show that

$$
\sum_{J \subset Q, J \in G_{k+1}}|J| \leq \frac{C}{A}|Q|\|f\|_{B M O_{\circ}(\mathbb{R})}
$$

For that, we write

$$
\sum_{J \subset Q, J \in G_{k+1}}|J| \leq \frac{1}{A} \sum_{J \subset Q, J \in G_{k+1}}|J| x_{J}^{\lambda}\left|x_{Q}^{-\lambda} u\left(x_{Q}, t_{Q}\right)-x_{J}^{-\lambda} u\left(x_{J}, t_{J}\right)\right|,
$$

and use the following decomposition for every $J \in G_{k+1}, J \subset Q$,

$$
\begin{aligned}
x_{Q}^{-\lambda} u\left(x_{Q}, t_{Q}\right)-x_{J}^{-\lambda} u\left(x_{J}, t_{J}\right)= & x_{Q}^{-\lambda} P_{t_{Q}}^{\lambda}\left(f-f_{Q}\right)\left(x_{Q}\right)-x_{J}^{-\lambda} P_{t_{J}}^{\lambda}\left(f-f_{J}\right)\left(x_{J}\right) \\
& +x_{Q}^{-\lambda} f_{Q}\left[P_{t_{J}}^{\lambda}(1)\left(x_{Q}\right)-1\right]-x_{J}^{-\lambda} f_{J}\left[P_{t_{J}}^{\lambda}(1)\left(x_{J}\right)-1\right] \\
& +\left[x_{Q}^{-\lambda} f_{Q}-x_{J}^{-\lambda} f_{J}\right] \\
= & : \sum_{i=1}^{5} H_{i}(J) .
\end{aligned}
$$

Let $J \in G_{k+1}, J \subset Q$. According to (3.7) and since $x_{J} \leq 2 x_{Q}$ we obtain

$$
x_{J}^{\lambda}\left|H_{1}(J)+H_{2}(J)\right| \leq C\left[\left(\frac{x_{J}}{x_{Q}}\right)^{\lambda}+1\right]\|f\|_{B M O_{\circ}(\mathbb{R})} \leq C\|f\|_{B M O_{\circ}(\mathbb{R})} .
$$

By using (3.8) and (3.11) and proceeding as in (3.9) and in (3.12) we also get

$$
x_{J}^{\lambda}\left|H_{3}(J)+H_{4}(J)\right| \leq C\left(\left|f_{Q} \| P_{t_{Q}}^{\lambda}(1)\left(x_{Q}\right)-1\right|+\left|f_{J}\right|\left|P_{t_{J}}^{\lambda}(1)\left(x_{J}\right)-1\right|\right) \leq C\|f\|_{B M O_{\circ}(\mathbb{R})} .
$$

We now study $H_{5}$. We can write

$$
x_{J}^{\lambda} H_{5} \leq\left|\left[\left(\frac{x_{J}}{x_{Q}}\right)^{\lambda}-1\right] f_{Q}\right|+\left|f_{Q}-f_{J}\right| .
$$


If $x_{Q} \leq t_{Q}$, then, as in (3.9),

$$
\left|\left[\left(\frac{x_{J}}{x_{Q}}\right)^{\lambda}-1\right] f_{Q}\right| \leq C\left|f_{Q}\right| \leq C\|f\|_{B M O_{\mathrm{o}}(\mathbb{R})} .
$$

In the case that $x_{Q}>t_{Q}$, since $J \subset Q$, it follows that $x_{J} / x_{Q} \subset(1 / 2,3 / 2)$, and then, by applying the mean value theorem we get

$$
\left|\left[\left(\frac{x_{J}}{x_{Q}}\right)^{\lambda}-1\right] f_{Q}\right| \leq C \frac{\left|x_{Q}-x_{J}\right|}{x_{Q}}\left|f_{Q}\right| \leq C \frac{t_{Q}}{x_{Q}}\left|f_{Q}\right| \leq C \frac{x_{Q}+t_{Q}}{x_{Q}}\|f\|_{B M O_{\circ}(\mathbb{R})} \leq C\|f\|_{B M O_{\circ}(\mathbb{R})} .
$$

By combining the above estimates we conclude that

$$
\begin{aligned}
\sum_{J \subset Q, J \in G_{k+1}}|J| & \leq \frac{C}{A} \sum_{J \subset Q, J \in G_{k+1}}|J|\left(\|f\|_{B M O_{\circ}(\mathbb{R})}+\left|f_{Q}-f_{J}\right|\right) \\
& \leq \frac{C}{A}\left(|Q|\|f\|_{B M O_{\circ}(\mathbb{R})}+\sum_{J \subset Q, J \in G_{k+1}} \int_{J}\left|f(y)-f_{Q}\right| d y\right) \\
& \leq \frac{C}{A}\left(|Q|\|f\|_{B M O_{\circ}(\mathbb{R})}+\int_{Q}\left|f(y)-f_{Q}\right| d y\right) \leq \frac{C}{A}|Q|\|f\|_{B M O_{\circ}(\mathbb{R})},
\end{aligned}
$$

and (4.3) is established.

By choosing $A:=2 C\left(1+\|f\|_{B M O_{\mathrm{o}}(\mathbb{R})}\right)$ we obtain

$$
\sum_{J \subset Q, J \in G_{k+1}}|J| \leq \frac{|Q|}{2}
$$

for every $Q \in G_{k}, k \in \mathbb{N}$.

Another helpful property is the following. Let $0 \leq a<b<\infty$ and $0<c<d<\infty$. For every $\alpha \in \mathbb{R}$, we have that

$$
\begin{array}{rl}
2 \int_{c}^{d} \int_{a}^{b} & t \nabla_{\lambda, y}\left(P_{t}^{\lambda}(x, y)\right) \cdot \nabla_{\lambda, y}\left(P_{t}^{\lambda}(f)(y)-\alpha y^{\lambda}\right) d y d t \\
= & \int_{a}^{b}\left[t \partial_{t}\left(P_{t}^{\lambda}(x, y)\right)\left(P_{t}^{\lambda}(f)(y)-\alpha y^{\lambda}\right)+t P_{t}^{\lambda}(x, y) \partial_{t}\left(P_{t}^{\lambda}(f)(y)\right)\right. \\
& \left.\quad-P_{t}^{\lambda}(x, y)\left(P_{t}^{\lambda}(f)(y)-\alpha y^{\lambda}\right)\right]_{t=c}^{t=d} d y \\
& +\int_{c}^{d} t\left[P_{t}^{\lambda}(x, y) D_{\lambda, y}\left(P_{t}^{\lambda}(f)(y)\right)+D_{\lambda, y}\left(P_{t}^{\lambda}(x, y)\right)\left(P_{t}^{\lambda}(f)(y)-\alpha y^{\lambda}\right)\right]_{y=a}^{y=b} d t \\
:= & \int_{a}^{b}\left[H_{\alpha}(x, y, t)\right]_{t=c}^{t=d} d y+\int_{c}^{d}\left[V_{\alpha}(x, y, t)\right]_{y=a}^{y=b} d t, \quad x \in(0, \infty) .
\end{array}
$$

Indeed, by integrating by parts we get, for all $x \in(0, \infty)$,

$$
\begin{aligned}
\int_{c}^{d} \int_{a}^{b} t D_{\lambda, y}\left(P_{t}^{\lambda}(x, y)\right) D_{\lambda, y}\left(P_{t}^{\lambda}(f)(y)\right) d y d t \\
=\frac{1}{2}\left\{\int_{c}^{d}\left[t P_{t}^{\lambda}(x, y) D_{\lambda, y}\left(P_{t}^{\lambda}(f)(y)\right)+t D_{\lambda, y}\left(P_{t}^{\lambda}(x, y)\right) P_{t}^{\lambda}(f)(y)\right]_{y=a}^{y=b} d t\right. \\
\left.\quad+\int_{c}^{d} \int_{a}^{b} t\left(P_{t}^{\lambda}(x, y) B_{\lambda, y}\left(P_{t}^{\lambda}(f)(y)\right)+B_{\lambda, y}\left(P_{t}^{\lambda}(x, y)\right) P_{t}^{\lambda}(f)(y)\right) d y d t\right\} .
\end{aligned}
$$

Also we have that

$$
\begin{aligned}
\int_{c}^{d} \int_{a}^{b} t \partial_{t}\left(P_{t}^{\lambda}(x, y)\right) \partial_{t}\left(P_{t}^{\lambda}(f)(y)\right) d y d t \\
=\frac{1}{2} \int_{c}^{d} \int_{a}^{b} t\left\{\partial_{t}^{2}\left[P_{t}^{\lambda}(x, y) P_{t}^{\lambda}(f)(y)\right]-\partial_{t}^{2}\left(P_{t}^{\lambda}(x, y)\right) P_{t}^{\lambda}(f)(y)\right. \\
\left.\quad \quad-P_{t}^{\lambda}(x, y) \partial_{t}^{2}\left(P_{t}^{\lambda}(f)(y)\right)\right\} d y d t, \quad x \in(0, \infty) .
\end{aligned}
$$

By [6, Lemma 2.2 and (2.12)]

$$
\left(\partial_{t}^{2}-B_{\lambda, y}\right) P_{t}^{\lambda}(f)(y)=0 \quad \text { and } \quad\left(\partial_{t}^{2}-B_{\lambda, y}\right) P_{t}^{\lambda}(x, y)=0, \quad t, x, y \in(0, \infty),
$$


we obtain, for $x \in(0, \infty)$,

$$
\begin{aligned}
\int_{c}^{d} \int_{a}^{b} t & \nabla_{\lambda, y}\left(P_{t}^{\lambda}(x, y)\right) \cdot \nabla_{\lambda, y}\left(P_{t}^{\lambda}(f)(y)\right) d y d t \\
= & \frac{1}{2}\left(\int_{a}^{b} \int_{c}^{d} t \partial_{t}^{2}\left[P_{t}^{\lambda}(x, y) P_{t}^{\lambda}(f)(y)\right] d t d y\right. \\
& \left.\quad+\int_{c}^{d}\left[t P_{t}^{\lambda}(x, y) D_{\lambda, y}\left(P_{t}^{\lambda}(f)(y)\right)+t D_{\lambda, y}\left(P_{t}^{\lambda}(x, y)\right) P_{t}^{\lambda}(f)(y)\right]_{y=a}^{y=b} d t\right) \\
=\frac{1}{2} & \left(\int_{a}^{b}\left[t \partial_{t}\left[P_{t}^{\lambda}(x, y) P_{t}^{\lambda}(f)(y)\right]-P_{t}^{\lambda}(x, y) P_{t}^{\lambda}(f)(y)\right]_{t=c}^{t=d} d y\right. \\
& \left.\quad+\int_{c}^{d}\left[t P_{t}^{\lambda}(x, y) D_{\lambda, y}\left(P_{t}^{\lambda}(f)(y)\right)+t D_{\lambda, y}\left(P_{t}^{\lambda}(x, y)\right) P_{t}^{\lambda}(f)(y)\right]_{y=a}^{y=b} d t\right) \\
= & \frac{1}{2}\left(\int_{a}^{b}\left[t \partial_{t}\left(P_{t}^{\lambda}(x, y)\right) P_{t}^{\lambda}(f)(y)+t P_{t}^{\lambda}(x, y) \partial_{t}\left(P_{t}^{\lambda}(f)(y)\right)-P_{t}^{\lambda}(x, y) P_{t}^{\lambda}(f)(y)\right]_{t=c}^{t=d} d y\right. \\
& \left.\quad+\int_{c}^{d}\left[t P_{t}^{\lambda}(x, y) D_{\lambda, y}\left(P_{t}^{\lambda}(f)(y)\right)+t D_{\lambda, y}\left(P_{t}^{\lambda}(x, y)\right) P_{t}^{\lambda}(f)(y)\right]_{y=a}^{y=b} d t\right) .
\end{aligned}
$$

Then, by taking into account that $\nabla_{\lambda, y}\left(\alpha y^{\lambda}\right)=(0,0)$, for every $\alpha \in \mathbb{R}$, we obtain (4.5).

In order to prove Theorem 1.1 (ii), by using (2.11), we can write

$$
f(x)=2 \lim _{n \rightarrow \infty} \int_{2^{-n}}^{2^{n}} \int_{0}^{\infty} t \nabla_{\lambda, y}\left(P_{t}^{\lambda}(x, y)\right) \cdot \nabla_{\lambda, y}\left(P_{t}^{\lambda}(f)(y)\right) d y d t, \quad \text { in } L^{2}(0, \infty) .
$$

For every $n \in \mathbb{N}$ we define the sets

$$
\begin{aligned}
U_{n} & =[0,2) \times\left(2^{-n}, 2\right), \\
W_{n} & =[0, \infty) \times\left(2^{-n}, 2^{n}\right) \backslash U_{n},
\end{aligned}
$$

and, for every $k \in \mathbb{N}$ and $Q \in G_{k}, \Sigma_{Q, n}:=\Sigma_{Q} \cap U_{n}$. Note that if $k, n \in \mathbb{N}$ and $k>n$, then $\Sigma_{Q, n}=\emptyset$, for each $Q \in G_{k}$.
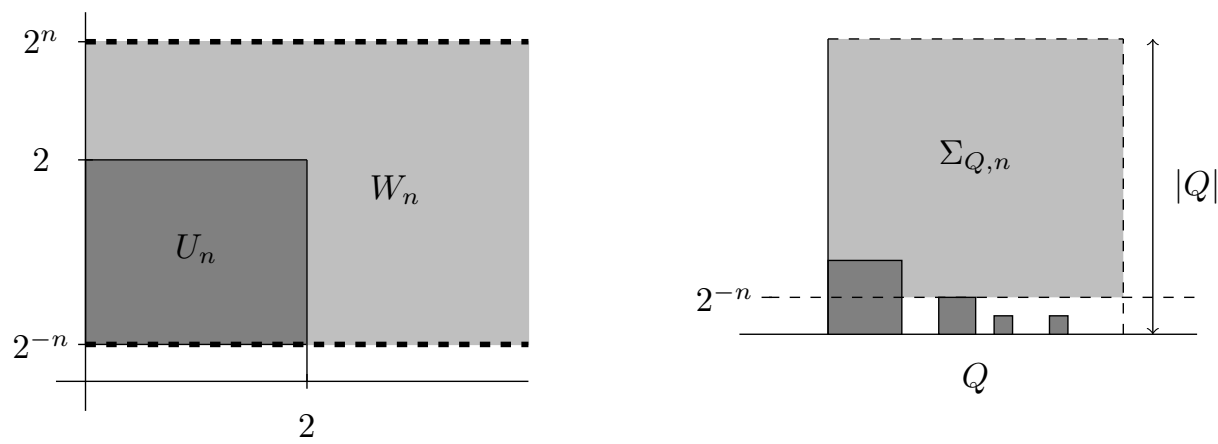

We obtain, for each $n \in \mathbb{N}$,

$$
\begin{aligned}
2 \int_{2^{-n}}^{2^{n}} \int_{0}^{\infty} t \nabla_{\lambda, y}\left(P_{t}^{\lambda}(x, y)\right) & \nabla_{\lambda, y}\left(P_{t}^{\lambda}(f)(y)\right) d y d t \\
= & 2\left(\int_{U_{n}}+\int_{W_{n}}\right) t \nabla_{\lambda, y}\left(P_{t}^{\lambda}(x, y)\right) \cdot \nabla_{\lambda, y}\left(P_{t}^{\lambda}(f)(y)\right) d y d t \\
= & 2\left(\int_{\bigcup_{Q \in \cup_{k=0}^{n} G_{k}} \Sigma_{Q, n}}+\int_{W_{n}}\right) t \nabla_{\lambda, y}\left(P_{t}^{\lambda}(x, y)\right) \cdot \nabla_{\lambda, y}\left(P_{t}^{\lambda}(f)(y)\right) d y d t \\
= & : G_{1, n}(x)+G_{2, n}(x), \quad x \in(0, \infty) .
\end{aligned}
$$

Our objective is to establish that, there exists an increasing sequence $\left\{n_{i}\right\}_{i \in \mathbb{N}}$ of nonnegative integers such that

$$
\lim _{i \rightarrow \infty} G_{1, n_{i}}(x)=g_{1}(x)+S_{\sigma_{1}, P^{\lambda}}(x), \quad \text { a.e. } x \in(0, \infty)
$$

$$
\lim _{i \rightarrow \infty} G_{2, n_{i}}(x)=g_{2}(x)+S_{\sigma_{2}, P^{\lambda}}(x), \quad \text { a.e. } x \in(0, \infty),
$$


for certain $g_{1}$ and $g_{2} \in L^{\infty}(0, \infty)$ and $\sigma_{1}$ and $\sigma_{2}$ Carleson measures on $(0, \infty) \times(0, \infty)$ such that

$$
\left\|g_{1}\right\|_{\infty}+\left\|g_{2}\right\|_{\infty}+\left\|\sigma_{1}\right\|_{\mathcal{C}}+\left\|\sigma_{2}\right\|_{\mathcal{C}} \leq C\left(A+\|f\|_{B M O_{\circ}(\mathbb{R})}\right),
$$

and thus we can conclude our result.

Let $n \in \mathbb{N}$. First we deal with the function $G_{1, n}$. We can write

$$
G_{1, n}(x)=\sum_{Q \in \cup_{k \in \mathbb{N}} G_{k}} 2 \int_{\Sigma_{Q, n}} t \nabla_{\lambda, y}\left(P_{t}^{\lambda}(x, y)\right) \cdot \nabla_{\lambda, y}\left[P_{t}^{\lambda}(f)(y)-c_{Q} y^{\lambda}\right] d y d t,
$$

where $c_{Q}:=y^{-\lambda} P_{t}^{\lambda}(f)(y)_{\left.\right|_{(y, t)=\left(x_{Q}, t_{Q}\right)}}$.

Let $k \in \mathbb{N}$ and $Q \in G_{k}$. By taking into account (4.5) it follows that the integral

$$
\int_{\Sigma_{Q, n}} t \nabla_{\lambda, y}\left(P_{t}^{\lambda}(x, y)\right) \cdot \nabla_{\lambda, y}\left[P_{t}^{\lambda}(f)(y)-c_{Q} y^{\lambda}\right] d y d t
$$

reduces to an integral over the boundary $\partial \Sigma_{Q, n}$ of $\Sigma_{Q, n}$.

We decompose this boundary in vertical and horizontal segments as follows. Let us denote by $\mathcal{V}_{Q, n}$ the set of vertical segments in $\partial \Sigma_{Q, n} \cap\left([0,2] \times\left[2^{-n}, 2\right]\right)$, by $\mathcal{H}_{Q, n}$ the set constituted by all horizontal segments in $\partial \Sigma_{Q, n} \cap\left([0,2] \times\left(2^{-n}, 2\right]\right)$ and those ones in $\partial \Sigma_{Q, n} \cap\left([0,2] \times\left\{2^{-n}\right\}\right)$ which belong to the boundary of some $Q^{\prime} \subset Q, Q^{\prime} \in G_{k+1}$ with $\left|Q^{\prime}\right|=2^{-n}$ and finally we consider $\mathcal{H}_{Q, n}^{0}$ the set of all horizontal segments in $\partial \Sigma_{Q, n} \cap\left([0,2] \times\left\{2^{-n}\right\}\right)$ that are not in $\mathcal{H}_{Q, n}$.

Indeed we can write

$$
\begin{aligned}
\mathcal{H}_{Q, n} & =\bigcup_{I \in \mathbb{I}_{Q, n}}(I \times\{|I|\}), \\
\mathcal{H}_{Q, n}^{0} & =\bigcup_{J \in \mathbb{I}_{Q, n}^{0}}\left(J \times\left\{2^{-n}\right\}\right), \\
\mathcal{V}_{Q, n} & =\bigcup_{K \in \mathbb{K}_{Q, n}}\left(\left\{a_{K}\right\} \times K\right),
\end{aligned}
$$

where, when $k \leq n, \mathbb{I}_{Q, n}$ is the set constituted by $Q$ and all intervals $I \subset Q, I \in G_{k+1}$ with $|I| \geq 2^{-n}, \mathbb{I}_{Q, n}^{0}$ contains the maximal dyadic intervals $J \subset Q \backslash\left\{I \subset Q, I \in G_{k+1},|I| \geq 2^{-n}\right\}, \mathbb{K}_{Q, n}$ is a finite set of dyadic intervals in $\left[2^{-n}, 2\right]$, and $a_{K} \in[0,2]$, for every $K \in \mathbb{K}_{Q, n}$. When $k>n$, we consider $\mathbb{I}_{Q, n}=\mathbb{I}_{Q, n}^{0}=\mathbb{K}_{Q, n}=\emptyset$.

According to (4.5) we have that

$$
\begin{aligned}
G_{1, n}(x)= & -\sum_{Q \in \cup_{k \in \mathbb{N}} G_{k}} \sum_{J \in \mathbb{I}_{Q, n}^{0}} \int_{J} H_{c_{Q}}(x, y, t)_{\mid t=2^{-n}} d y \\
& +\sum_{Q \in \cup_{k \in \mathbb{N}} G_{k}}\left(\sum_{I \in \mathbb{I}_{Q, n}} \varepsilon_{I} \int_{I} H_{c_{Q}}(x, y, t)_{|t=| I \mid} d y+\sum_{K \in \mathbb{K}_{Q, n}} \varepsilon_{K} \int_{K} V_{c_{Q}}(x, y, t)_{\mid y=a_{K}} d t\right) \\
:= & \mathfrak{g}_{1, n}(x)+\mathfrak{g}_{2, n}(x), \quad x \in(0, \infty) .
\end{aligned}
$$

Here $\varepsilon_{J}= \pm 1, J \in \mathbb{I}_{Q, n} \cup \mathbb{K}_{Q, n}$.

Next we show that

$$
\lim _{n \rightarrow \infty} \mathfrak{g}_{1, n}(x)=\sum_{Q \in \cup_{k \in \mathbb{N}} G_{k}}\left(f(x)-c_{Q} x^{\lambda}\right) \chi_{\partial \Sigma_{Q} \cap([0,2] \times\{0\})}(x)=: g_{1}(x),
$$

in $L^{2}(0, \infty)$.

We can write, for every $n \in \mathbb{N}$,

$$
\begin{aligned}
\mathfrak{g}_{1, n}(x)= & -\int_{0}^{\infty} \sum_{Q \in \cup_{\in \mathbb{N}} G_{k}} \chi_{\mathbb{I}_{Q, n}^{0}}(y) H_{c_{Q}}(x, y, t)_{\mid t=2^{-n}} d y \\
= & \int_{0}^{\infty}\left(P_{t}^{\lambda}(x, y) \sum_{Q \in \cup_{k \in \mathbb{N} G_{k}}} \chi_{\mathbb{I}_{Q, n}^{0}}(y)\left[P_{t}^{\lambda}(f)(y)-c_{Q} y^{\lambda}\right]\right)_{\mid t=2^{-n}} d y \\
& -\int_{0}^{\infty}\left(t \partial_{t} P_{t}^{\lambda}(x, y) \sum_{Q \in \cup_{k \in \mathbb{N} G_{k}}} \chi_{\mathbb{I}_{Q, n}^{0}}(y)\left[P_{t}^{\lambda}(f)(y)-c_{Q} y^{\lambda}\right]\right)_{\mid t=2^{-n}} d y
\end{aligned}
$$




$$
-\int_{0}^{\infty}\left(P_{t}^{\lambda}(x, y) \sum_{Q \in \cup_{k \in \mathbb{N}} G_{k}} \chi_{\mathbb{I}_{Q, n}^{0}}(y) t \partial_{t}\left(P_{t}^{\lambda}(f)(y)\right)\right)_{\mid t=2^{-n}} d y, \quad x \in(0, \infty) .
$$

According to (4.2), for $k \in \mathbb{N}$ and $Q \in G_{k}$,

(4.10) $\left|P_{t}^{\lambda}(f)(y)-c_{Q} y^{\lambda}\right|=y^{\lambda}\left|y^{-\lambda} u(y, t)-x_{Q}^{-\lambda} u\left(x_{Q}, t_{Q}\right)\right| \leq C\left(A+\|f\|_{B M O_{o}(\mathbb{R})}\right), \quad(y, t) \in \Sigma_{Q}$.

By using (2.10), since $f \in L^{2}(0, \infty)$,

$$
\lim _{t \rightarrow 0^{+}} P_{t}^{\lambda}(f)(y)=f(y), \quad \text { a.e. } y \in(0, \infty) \text {. }
$$

Then it follows that

$$
\left|f(y)-c_{Q} y^{\lambda}\right| \leq C\left(A+\|f\|_{B M O_{\circ}(\mathbb{R})}\right), \quad \text { a.e. } y \in \partial \Sigma_{Q} \cap([0,2] \times\{0\}) .
$$

We observe also that

$$
\operatorname{supp}\left(\sum_{Q \in \cup_{\in \mathbb{N}} G_{k}} \chi_{\mathbb{I}_{Q, n}^{0}}(y)\left[P_{t}^{\lambda}(f)(y)-c_{Q} y^{\lambda}\right]_{\mid t=2^{-n}}-g_{1}(y)\right) \subset[0,2], \quad n \in \mathbb{N},
$$

and

$$
\lim _{n \rightarrow \infty} \sum_{Q \in \cup_{k \in \mathbb{N} G_{k}}} \chi_{\mathbb{I}_{Q, n}^{0}}(y)\left[P_{t}^{\lambda}(f)(y)-c_{Q} y^{\lambda}\right]_{\mid t=2^{-n}}=g_{1}(y), \quad \text { a.e. } y \in(0, \infty),
$$

(actually, we can assure that (4.12) is true for all $y \in Q_{0}$ which is not a dyadic number). By the dominated convergence theorem, we get (4.12) in $L^{2}(0, \infty)$.

According to (2.5) and (2.6) we have, for each $n \in \mathbb{N}$,

$$
\begin{aligned}
\mathfrak{g}_{1, n}(x)-g_{1}(x)= & h_{\lambda}\left(e^{-t z} h_{\lambda}\left(\sum_{Q \in \cup_{\in \mathbb{N}} G_{k}} \chi_{\mathbb{I}_{Q, n}^{0}}(y)\left[P_{t}^{\lambda}(f)(y)-c_{Q} y^{\lambda}\right]-g_{1}(y)\right)(z)_{\mid t=2^{-n}}\right)(x) \\
& +h_{\lambda}\left(\left(e^{-t z}-1\right)_{\mid t=2^{-n}} h_{\lambda}\left(g_{1}\right)(z)\right)(x) \\
& +h_{\lambda}\left(t z e^{-t z} h_{\lambda}\left(\sum_{Q \in \cup_{k \in \mathbb{N} G_{k}}} \chi_{\mathbb{I}_{Q, n}^{0}}(y)\left[P_{t}^{\lambda}(f)(y)-c_{Q} y^{\lambda}\right]-g_{1}(y)\right)(z)_{\mid t=2^{-n}}\right)(x) \\
& -\left(t \partial_{t} P_{t}^{\lambda}\left(g_{1}\right)(x)\right)_{\mid t=2^{-n}} \\
& -h_{\lambda}\left(e^{-t z} h_{\lambda}\left(\sum_{Q \in \cup_{k \in \mathbb{N}} G_{k}} \chi_{\mathbb{I}_{Q, n}^{0}}(y) t \partial_{t}\left(P_{t}^{\lambda}(f)(y)\right)\right)(z)_{\mid t=2^{-n}}\right)(x), \quad x \in(0, \infty) .
\end{aligned}
$$

Then, by taking into account the $L^{2}$-boundedness of $h_{\lambda}$ we get

$$
\begin{aligned}
\left\|\mathfrak{g}_{1, n}-g_{1}\right\|_{2} \leq & C\left(\left\|\sum_{Q \in \cup_{k \in \mathbb{N} G_{k}}} \chi_{\mathbb{I}_{Q, n}^{0}}(y)\left[P_{t}^{\lambda}(f)(y)-c_{Q} y^{\lambda}\right]_{\mid t=2^{-n}}-g_{1}(y)\right\|_{2}\right. \\
& +\left\|\left(e^{-t z}-1\right)_{\mid t=2^{-n}} h_{\lambda}\left(g_{1}\right)\right\|_{2}+\left\|\left(t \partial_{t} P_{t}^{\lambda}\left(g_{1}\right)\right)_{\mid t=2^{-n}}\right\|_{2} \\
& \left.+\left\|\left(t \partial_{t} P_{t}^{\lambda}(f)\right)_{t=2^{-n}}\right\|_{2}\right), \quad n \in \mathbb{N} .
\end{aligned}
$$

From (2.9), (4.12) and the dominated convergence theorem we obtain (4.9). Note that by (4.11) we have that $\left\|g_{1}\right\|_{\infty} \leq C\left(A+\|f\|_{B M O_{\circ}(\mathbb{R})}\right)$.

Now, let us show that there exists a Carleson measure $\sigma_{1}$ on $(0, \infty)^{2}$ such that

$$
\lim _{n \rightarrow \infty} \mathfrak{g}_{2, n}(x)=S_{\sigma_{1}, P^{\lambda}}(x), \quad x \in(0, \infty) .
$$

For that, we write, for each $n \in \mathbb{N}$,

$$
\begin{aligned}
\mathfrak{g}_{2, n}(x)= & \sum_{Q \in \cup_{k \in \mathbb{N}} G_{k}}\left(\sum_{I \in \mathcal{H}_{Q, n}} \int_{I} P_{t}^{\lambda}(x, y) M_{Q, 1}(y, t) d y_{I}+\sum_{J \in \mathcal{V}_{Q, n}} \int_{J} P_{t}^{\lambda}(x, y) L_{Q, 1}(y, t) d t_{J}\right) \\
& +\sum_{Q \in \cup_{k \in \mathbb{N}} G_{k}}\left(\sum_{I \in \mathcal{H}_{Q, n}} \int_{I} M_{Q, 2}(x, y, t) d y_{I}+\sum_{J \in \mathcal{V}_{Q, n}} \int_{J} L_{Q, 2}(x, y, t) d t_{J}\right) \\
:= & F_{1, n}(x)+F_{2, n}(x), \quad x \in(0, \infty),
\end{aligned}
$$

where, for every $Q \in \cup_{k \in \mathbb{N}} G_{k}$,

$$
\begin{aligned}
& M_{Q, 1}(y, t):=t \partial_{t} P_{t}^{\lambda}(f)(y)-\left[P_{t}^{\lambda}(f)(y)-c_{Q} y^{\lambda}\right], \\
& L_{Q, 1}(y, t):=t D_{\lambda, y} P_{t}^{\lambda}(f)(y),
\end{aligned}
$$




$$
M_{Q, 2}(x, y, t):=t \partial_{t}\left(P_{t}^{\lambda}(x, y)\right)\left[P_{t}^{\lambda}(f)(y)-c_{Q} y^{\lambda}\right]
$$

and

Let us consider

$$
L_{Q, 2}(x, y, t):=t D_{\lambda, y} P_{t}^{\lambda}(x, y)\left[P_{t}^{\lambda}(f)(y)-c_{Q} y^{\lambda}\right] .
$$

$$
\mathcal{H}_{Q}:=\left\{\text { horizontal segments in } \partial \Sigma_{Q} \cap([0,2] \times(0,2])\right\},
$$

and

$$
\mathcal{V}_{Q}:=\left\{\text { vertical segments in } \partial \Sigma_{Q}\right\}
$$

By (4.4) and according to [16, p. 346] the measures

$$
\nu:=\sum_{Q \in \cup_{k \in \mathbb{N}} G_{k}}\left(\sum_{I \in \mathcal{H}_{Q}} d y_{I}+\sum_{J \in \mathcal{V}_{Q}} d t_{J}\right),
$$

and

$$
\nu_{n}:=\sum_{Q \in \cup_{k \in \mathbb{N}} G_{k}}\left(\sum_{I \in \mathcal{H}_{Q, n}} d y_{I}+\sum_{J \in \mathcal{V}_{Q, n}} d t_{J}\right), \quad n \in \mathbb{N},
$$

are Carleson measures. Moreover, we can write

$$
d \nu_{n}(y, t)=k_{n}(y, t) d \alpha(y, t) \text { and } d \nu(y, t)=k(y, t) d \alpha(y, t),
$$

for certain positive measure $\alpha$ and nonnegative functions $k_{n}$ and $k$ such that $k_{n} \uparrow k$, as $n \rightarrow \infty$, pointwisely. Then, $\left\|\nu_{n}\right\|_{\mathcal{C}} \leq\|\nu\|_{\mathcal{C}}, n \in \mathbb{N}$, and by the monotone convergence theorem,

$$
\lim _{n \rightarrow \infty} S_{\nu_{n}, P^{\lambda}}(x)=S_{\nu, P^{\lambda}}(x), \quad x \in(0, \infty) .
$$

We now define

$$
\mu_{n}(y, t):=\sum_{Q \in \cup_{k \in \mathbb{N}} G_{k}}\left(\sum_{I \in \mathcal{H}_{Q, n}} M_{Q, 1}(y, t) d y_{I}+\sum_{J \in \mathcal{V}_{Q, n}} L_{Q, 1}(y, t) d t_{J}\right), \quad n \in \mathbb{N},
$$

and

$$
\mu(y, t):=\sum_{Q \in \cup_{k \in \mathbb{N}} G_{k}}\left(\sum_{I \in \mathcal{H}_{Q}} M_{Q, 1}(y, t) d y_{I}+\sum_{J \in \mathcal{V}_{Q}} L_{Q, 1}(y, t) d t_{J}\right) .
$$

From Proposition 3.3 and (4.10) it follows that, for every $Q \in \bigcup_{k=0}^{\infty} G_{k}$,

$$
\left|M_{Q, 1}(y, t)\right| \leq C\left(A+\|f\|_{B M O_{\circ}(\mathbb{R})}\right), \quad(y, t) \in \Sigma_{Q},
$$

and

$$
\left|L_{Q, 1}(y, t)\right| \leq C\|f\|_{B M O_{o}(\mathbb{R})}, \quad y, t \in(0, \infty) .
$$

Then the measures $\mu$ and $\mu_{n}, n \in \mathbb{N}$, are Carleson measures, satisfying that

$$
\begin{gathered}
\left\|\mu_{n}\right\|_{\mathcal{C}} \leq C\left(A+\|f\|_{B M O_{\circ}(\mathbb{R})}\right), \quad n \in \mathbb{N}, \\
\|\mu\|_{\mathcal{C}} \leq C\left(A+\|f\|_{B M O_{\circ}(\mathbb{R})}\right),
\end{gathered}
$$

and, by Theorem 1.1 (i) and the dominated convergence theorem

$$
\lim _{n \rightarrow \infty} S_{\mu_{n}, P^{\lambda}}(x)=S_{\mu, P^{\lambda}}(x), \quad x \in(0, \infty) .
$$

Note that, for every $n \in \mathbb{N}, F_{1, n}(x)=S_{\mu_{n}, P^{\lambda}}(x), x \in(0, \infty)$. Then,

$$
\lim _{n \rightarrow \infty} F_{1, n}(x)=S_{\mu, P^{\lambda}}(x), \quad x \in(0, \infty) .
$$

Now we study $F_{2, n}, n \in \mathbb{N}$. By Lemma 2.1 and (2.3) we get

$$
\left|t \partial_{t} P_{t}^{\lambda}(x, y)\right|+\left|t D_{\lambda, y} P_{t}^{\lambda}(x, y)\right| \leq C \frac{t}{(x-y)^{2}+t^{2}}, \quad x, y, t \in(0, \infty) .
$$

Then, by taking into account (4.10) and proceeding as in [29, p. 25 and 26] (see also [8, p. 2088]), we get, for every $n \in \mathbb{N}$, a Carleson measure $\rho_{n}$ such that $F_{2, n}=S_{\rho_{n}, P^{\lambda}}$, and a Carleson measure $\rho$, such that

$$
\sum_{Q \in \cup_{k \in \mathbb{N}} G_{k}}\left(\sum_{I \in \mathcal{H}_{Q}} \int_{I} M_{Q, 2}(x, y, t) d y_{I}+\sum_{J \in \mathcal{V}_{Q}} \int_{J} L_{Q, 2}(x, y, t) d t_{J}\right)=S_{\rho, P^{\lambda}}(x), \quad x \in(0, \infty) .
$$

We have that,

$$
\lim _{n \rightarrow \infty} S_{\rho_{n}, P^{\lambda}}(x)=S_{\rho, P^{\lambda}}(x), \quad x \in(0, \infty),
$$


and $\|\rho\|_{\mathcal{C}} \leq C\left(A+\|f\|_{B M O_{\mathrm{o}}(\mathbb{R})}\right)$. Then we obtain (4.13) for $\sigma_{1}=\mu+\rho$. From (4.8), (4.9) and (4.13) we can find an increasing sequence $\left\{n_{i}\right\}_{i \in \mathbb{N}}$ of nonnegative integers such that

$$
\lim _{i \rightarrow \infty} G_{1, n_{i}}(x)=g_{1}(x)+S_{\mu+\rho, P^{\lambda}}(x), \quad \text { a.e. } x \in(0, \infty) .
$$

Note that $\left\|g_{1}\right\|_{\infty}+\left\|\sigma_{1}\right\|_{\mathcal{C}} \leq C\left(A+\|f\|_{B M O_{\circ}(\mathbb{R})}\right)$, so (4.6) is thus established.

We now deal with $G_{2, n}, n \in \mathbb{N}$. Let $M>2$. We define

$$
W_{n, M}:=\left\{(x, t) \in W_{n}: x \in(0, M)\right\}, \quad n \in \mathbb{N} .
$$

By (4.5) we have that

$$
\begin{aligned}
G_{2, n, M}(x):= & 2 \int_{W_{n, M}} t \nabla_{\lambda, y}\left(P_{t}^{\lambda}(x, y)\right) \cdot \nabla_{\lambda, y}\left[P_{t}^{\lambda}(f)(y)-c_{0} y^{\lambda}\right] d y d t \\
= & \int_{0}^{M} H_{c_{0}}(x, y, t)_{\mid t=2^{n}} d y-\int_{2}^{M} H_{c_{0}}(x, y, t)_{\mid t=2^{-n}} d y-\int_{0}^{2} H_{c_{0}}(x, y, t)_{\mid t=2} d y \\
& -\int_{2}^{2^{n}} V_{c_{0}}(x, y, t)_{\mid y=0} d t-\int_{2^{-n}}^{2} V_{c_{0}}(x, y, t)_{\mid y=2} d t+\int_{2^{-n}}^{2^{n}} V_{c_{0}}(x, y, t)_{\mid y=M} d t \\
= & : \sum_{i=1}^{6} I_{i, n, M}(x), \quad x \in(0, \infty) \text { and } n \in \mathbb{N},
\end{aligned}
$$

where $c_{0}:=x_{Q_{0}}^{-\lambda} u\left(x_{Q_{0}}, t_{Q_{0}}\right)$. Observe that, actually $I_{3, n, M}$ is independent of $n$ and $M$ and $I_{4, n, M}$ and $I_{5, n, M}$ do not depend on $M$.

First, we note that $I_{4, n, M}(x)=0, n \in \mathbb{N}$. Indeed, by Lemma 2.1 and (2.2) it follows that

$$
\lim _{y \rightarrow 0^{+}} P_{t}^{\lambda}(x, y)=\lim _{y \rightarrow 0^{+}} D_{\lambda, y} P_{t}^{\lambda}(x, y)=0, \quad x, t \in(0, \infty) \text {. }
$$

Then, by taking into account Proposition 3.3 and (4.10) for $Q=Q_{0}$ it follows that $V_{c_{0}}(x, y, t)_{\mid y=0}=$ $0, x, t \in(0, \infty)$.

On the other hand, we have that

$$
\begin{aligned}
I_{3, n, M}(x) & =-\int_{0}^{2}\left(P_{t}^{\lambda}(x, y) M_{Q_{0}, 1}(y, t)\right)_{\mid t=2} d y-\int_{0}^{2} M_{Q_{0}, 2}(y, t)_{\mid t=2} d y \\
& :=I_{3}^{1}(x)+I_{3}^{2}(x), \quad x \in(0, \infty) .
\end{aligned}
$$

Here $M_{Q_{0}, 1}$ and $M_{Q_{0}, 2}$ are as in (4.14) with $Q=Q_{0}$. By considering Proposition 3.3 and (4.10) we get a Carleson measure $\alpha_{3}^{1}$ such that $I_{3}^{1}(x)=S_{\alpha_{3}^{1}, P^{\lambda}}(x), x \in(0, \infty)$ and $\left\|\alpha_{3}^{1}\right\|_{\mathcal{C}} \leq C(A+$ $\|f\|_{B M O_{\circ}(\mathbb{R})}$ ). Also, from Lemma 2.1, (2.3) and (4.10) we deduce as above (see [29, p. 25 and 26]) that $I_{3}^{2}(x)=S_{\alpha_{3}^{2}, P^{\lambda}}(x), x \in(0, \infty)$, for some Carleson measure $\alpha_{3}^{2}$ such that $\left\|\alpha_{3}^{2}\right\|_{\mathcal{C}} \leq$ $C\left(A+\|f\|_{B M O_{\circ}(\mathbb{R})}\right)$. Then there exists a Carleson measure $\alpha_{3}=\alpha_{3}^{1}+\alpha_{3}^{2}$ on $(0, \infty)^{2}$ such that $I_{3, n, M}(x)=S_{\alpha_{3}, P^{\lambda}}(x), x \in(0, \infty)$.

In a similar way we can see that, for every $n \in \mathbb{N}$, there exists a Carleson measure $\alpha_{5, n}$ such that $I_{5, n, M}=S_{\alpha_{5, n}, P^{\lambda}}$ and $\left\|\alpha_{5, n}\right\|_{\mathcal{C}} \leq C\left(A+\|f\|_{B M O_{\mathrm{o}}(\mathbb{R})}\right)$. And, as above, by Theorem 1.1 and the dominated convergence theorem, there exists $\alpha_{5} \in \mathcal{C}$ such that $S_{\alpha_{5, n}, P^{\lambda}}(x) \rightarrow S_{\alpha_{5}, P^{\lambda}}(x)$, as $n \rightarrow \infty$, for a.e. $x \in(0, \infty)$.

We have also that, for every $n \in \mathbb{N}$,

$$
\lim _{M \rightarrow \infty} I_{6, n, M}(x)=0, \quad x \in(0, \infty) .
$$

It is sufficient to note that by Lemma 2.1 and (2.2) it follows that

$$
\begin{aligned}
\left|I_{6, n, M}(x)\right| & \leq C M^{\lambda} \int_{2^{-n}}^{2^{n}} \frac{t(x M)^{\lambda}}{\left((x-M)^{2}+t^{2}\right)^{\lambda+1}}\left(1+\frac{1}{t^{2 \lambda+2}} \int_{0}^{1}|f(z)| d z\right) d t \\
& \leq C \frac{x^{\lambda} M^{2 \lambda}}{\left(|x-M|+2^{-n}\right)^{2 \lambda+1}} \int_{2^{-n}}^{2^{n}}\left(1+\frac{1}{t^{2 \lambda+2}}\right) d t \leq \frac{C_{n, x} M^{2 \lambda}}{\left(|x-M|+2^{-n}\right)^{2 \lambda+1}}, \quad x \in(0, \infty),
\end{aligned}
$$

for every $n \in \mathbb{N}$ and for certain $C_{n, x}>0$.

On the other hand, for each $n \in \mathbb{N}$,

$$
\lim _{M \rightarrow \infty} I_{1, n, M}(x)=\int_{0}^{\infty} H_{c_{0}}(x, y, t)_{\mid t=2^{n}} d y, \quad x \in(0, \infty),
$$


and

$$
\lim _{M \rightarrow \infty} I_{2, n, M}(x)=\int_{2}^{\infty} H_{c_{0}}(x, y, t)_{\mid t=2^{-n}} d y, \quad x \in(0, \infty) .
$$

It is sufficient to show that for each $n \in \mathbb{N}$, the integrals in the right side of (4.15) and (4.16) are absolutely convergent for every $x \in(0, \infty)$. Indeed, from Lemma 2.1 and (2.2) and Proposition 3.3. we get

$$
\left|H_{c_{0}}(x, y, t)\right| \leq C \frac{t(x y)^{\lambda}}{\left((x-y)^{2}+t^{2}\right)^{\lambda+1}}\left(\left|P_{t}^{\lambda}(f)(y)\right|+y^{\lambda}+1\right), \quad x, y, t \in(0, \infty) .
$$

Since $f \in L^{2}(0, \infty)$, also $P_{t}^{\lambda}(f) \in L^{2}(0, \infty)$ and, by Hölder's inequality we deduce that

$$
\begin{aligned}
\int_{0}^{\infty}\left|H_{c_{0}}(x, y, t)\right| d y \leq C t x^{\lambda} & \int_{0}^{\infty} \frac{y^{\lambda}}{\left((x-y)^{2}+t^{2}\right)^{\lambda+1}}\left(\left|P_{t}^{\lambda}(f)(y)\right|+y^{\lambda}+1\right) d y \\
\leq C t x^{\lambda}\{ & \left(\int_{0}^{\infty} \frac{y^{2 \lambda}}{\left((x-y)^{2}+t^{2}\right)^{2 \lambda+2}} d y\right)^{1 / 2}\left\|P_{t}^{\lambda}(f)\right\|_{2} \\
& \left.+\int_{0}^{\infty} \frac{y^{\lambda}+y^{2 \lambda}}{\left((x-y)^{2}+t\right)^{\lambda+1}} d y\right\}<\infty, \quad x, t \in(0, \infty) .
\end{aligned}
$$

We conclude that, for every $n \in \mathbb{N}$,

$$
\begin{aligned}
G_{2, n}(x)= & \int_{0}^{\infty} H_{c_{0}}(x, y, t)_{\mid t=2^{n}} d y-\int_{2}^{\infty} H_{c_{0}}(x, y, t)_{\mid t=2^{-n}} d y \\
& +S_{\alpha_{3}, P^{\lambda}}(x)+S_{\alpha_{5, n}, P^{\lambda}}(x), \quad x \in(0, \infty) .
\end{aligned}
$$

In order to finish the proof we are going to show that

$$
\lim _{n \rightarrow \infty} \int_{0}^{\infty} H_{c_{0}}(x, y, t)_{\mid t=2^{n}} d y=c_{0} x^{\lambda}, \quad x \in(0, \infty),
$$

and

$$
\lim _{i \rightarrow \infty} \int_{2}^{\infty} H_{c_{0}}(x, y, t)_{\mid t=2^{-n_{i}}}=c_{0} x^{\lambda} \chi_{(2, \infty)}(x), \quad \text { a.e. } x \in(0, \infty),
$$

for certain increasing sequence $\left\{n_{i}\right\}_{i \in \mathbb{N}}$ of nonnegative integers. Thus we obtain that

$$
\lim _{i \rightarrow \infty} G_{2, n_{i}}(x)=c_{0} x^{\lambda} \chi_{(0,2)}(x)+S_{\alpha_{3}+\alpha_{5}, P^{\lambda}}(x), \quad \text { a.e. } x \in(0, \infty),
$$

and we get (4.7) with $g_{2}(x)=c_{0} x^{\lambda} \chi_{(0,2)}(x), x \in(0, \infty)$, and $\sigma_{2}=\alpha_{3}+\alpha_{5}$.

Since $P_{t}^{\lambda}\left(y^{\lambda}\right)(x)=x^{\lambda}, x, t \in(0, \infty)([1$, p. 455]),

$$
\int_{0}^{\infty} \partial_{t} P_{t}^{\lambda}(x, y) y^{\lambda} d y=\partial_{t} \int_{0}^{\infty} P_{t}^{\lambda}(x, y) y^{\lambda} d y=0, \quad x, t \in(0, \infty) .
$$

The derivation under the integral sign is justified because by Lemma 2.1 and (2.2) it follows that

$$
\int_{0}^{\infty}\left|\partial_{t} P_{t}^{\lambda}(x, y) y^{\lambda}\right| d y \leq C \int_{0}^{\infty} \frac{x^{\lambda} y^{2 \lambda}}{\left((x-y)^{2}+t^{2}\right)^{\lambda+1}} d y<\infty, \quad x, t \in(0, \infty) .
$$

Then, we can write

$$
\begin{aligned}
\int_{0}^{\infty} H_{c_{0}}(x, y, t) d y= & \int_{0}^{\infty}\left(t \partial_{t}\left(P_{t}^{\lambda}(x, y)\right) P_{t}^{\lambda}(f)(y)+t P_{t}^{\lambda}(x, y) \partial_{t} P_{t}^{\lambda}(f)(y)-P_{t}^{\lambda}(x, y) P_{t}^{\lambda}(f)(y)\right) d y \\
& +c_{0} x^{\lambda}, \quad x, t \in(0, \infty) .
\end{aligned}
$$

From Lemma 2.1 and (2.3) we have that

$$
\begin{aligned}
\left|\int_{0}^{\infty}\left(t \partial_{t}\left(P_{t}^{\lambda}(x, y)\right) P_{t}^{\lambda}(f)(y)+t P_{t}^{\lambda}(x, y) \partial_{t} P_{t}^{\lambda}(f)(y)-P_{t}^{\lambda}(x, y) P_{t}^{\lambda}(f)(y)\right) d y\right| \\
\leq C \int_{0}^{\infty} \frac{t}{(x-y)^{2}+t^{2}} \int_{0}^{1} \frac{t}{(y-z)^{2}+t^{2}}|f(z)| d z d y \\
\leq \frac{C}{t} \int_{0}^{\infty} \frac{t}{(x-y)^{2}+t^{2}} \int_{0}^{1}|f(z)| d z d y \\
\leq C \frac{\|f\|_{B M O_{o}(\mathbb{R})}}{t}\left(\int_{0}^{2 x} \frac{d y}{t}+\int_{2 x}^{\infty} \frac{t}{(y+t)^{2}} d y\right)
\end{aligned}
$$




$$
\leq \frac{C}{t}\left(\frac{x}{t}+\frac{t}{x+t}\right), \quad x, t \in(0, \infty) .
$$

Note that, for each $x \in(0, \infty)$, the last term tends to zero as $t \rightarrow \infty$, and consequently we get (4.17).

On the other hand, we have that

$$
\begin{aligned}
\int_{2}^{\infty} H_{c_{0}}(x, y, t) d y= & \int_{2}^{\infty}\left(t \partial_{t}\left(P_{t}^{\lambda}(x, y)\right) P_{t}^{\lambda}(f)(y)+t P_{t}^{\lambda}(x, y) \partial_{t} P_{t}^{\lambda}(f)(y)-P_{t}^{\lambda}(x, y) P_{t}^{\lambda}(f)(y)\right) d y \\
& -c_{0} t \partial_{t} \int_{2}^{\infty} P_{t}^{\lambda}(x, y) y^{\lambda} d y+c_{0} \int_{2}^{\infty} P_{t}^{\lambda}(x, y) y^{\lambda} d y \\
= & \int_{2}^{\infty}\left(t \partial_{t}\left(P_{t}^{\lambda}(x, y)\right) P_{t}^{\lambda}(f)(y)+t P_{t}^{\lambda}(x, y) \partial_{t} P_{t}^{\lambda}(f)(y)-P_{t}^{\lambda}(x, y) P_{t}^{\lambda}(f)(y)\right) d y \\
& +c_{0} t \partial_{t} P_{t}^{\lambda}\left(y^{\lambda} \chi_{(0,2)}(y)\right)(x)+c_{0} x^{\lambda}-c_{0} P_{t}^{\lambda}\left(y^{\lambda} \chi_{(0,2)}(y)\right)(x), \quad x, t \in(0, \infty)
\end{aligned}
$$

As above, by using Lemma 2.1 and (2.2) it follows that

$$
\begin{aligned}
\left|\int_{2}^{\infty}\left(t \partial_{t}\left(P_{t}^{\lambda}(x, y)\right) P_{t}^{\lambda}(f)(y)+t P_{t}^{\lambda}(x, y) \partial_{t} P_{t}^{\lambda}(f)(y)-P_{t}^{\lambda}(x, y) P_{t}^{\lambda}(f)(y)\right) d y\right| \\
\leq C \int_{2}^{\infty} P_{t}^{\lambda}(x, y) \int_{0}^{1} \frac{t(y z)^{\lambda}}{\left((y-z)^{2}+t^{2}\right)^{\lambda+1}}|f(z)| d z d y \\
\leq C t \int_{2}^{\infty} P_{t}^{\lambda}(x, y) y^{\lambda} \int_{0}^{1} \frac{|f(z)|}{\left(1+t^{2}\right)^{\lambda+1}} d z d y \\
\leq C\|f\|_{B M O_{\circ}(\mathbb{R})} t \int_{0}^{\infty} P_{t}^{\lambda}(x, y) y^{\lambda} d y \\
\leq C x^{\lambda} t, \quad x, t \in(0, \infty) .
\end{aligned}
$$

Observe that, for each $x \in(0, \infty)$, the last term tends to zero as $t \rightarrow 0$. By taking into account (2.9) and (2.10) we conclude that, there exists an increasing sequence $\left\{n_{i}\right\}_{i \in \mathbb{N}}$ of nonnegative integers such that

$$
\lim _{i \rightarrow \infty} \int_{2}^{\infty} H_{c_{0}}(x, y, t)_{\mid t=2^{-n_{i}}} d y=c_{0} x^{\lambda}\left(1-\chi_{(0,2)}(x)\right)=c_{0} x^{\lambda} \chi_{(2, \infty)}(x), \quad \text { a.e. } x \in(0, \infty),
$$

and (4.18) is proved.

\section{REFERENCES}

[1] J. J. Betancor, A. J. Castro, J. C. Fariña, and L. Rodríguez-Mesa, Solutions of Weinstein equations representable by Bessel Poisson integrals of BMO functions, J. Math. Anal. Appl., 431 (2015), pp. 440-470.

[2] J. J. Betancor, A. J. Castro, and P. R. Stinga, The fractional Bessel equation in Hölder spaces, J. Approx. Theory, 184 (2014), pp. 55-99.

[3] J. J. Betancor, A. Chicco Ruiz, J. C. Fariña, and L. Rodríguez-Mesa, Odd BMO(R) functions and Carleson measures in the Bessel setting, Integral Equations Operator Theory, 66 (2010), pp. 463-494.

[4] J. J. Betancor, X. T. Duong, J. Li, B. W. Wick, And D. Yang, Product Hardy, BMO spaces and iterated commutators associated with Bessel Schrödinger operators. To appear in Indiana Univ. Math. J. (available at arXiv:1701.05161).

[5] J. J. Betancor, J. Dziubański, and J. L. Torrea, On Hardy spaces associated with Bessel operators, J. Anal. Math., 107 (2009), pp. 195-219.

[6] J. J. Betancor and K. Stempak, On Hankel conjugate functions, Studia Sci. Math. Hungar., 41 (2004), pp. 59-91.

[7] L. Carleson, Two remarks on $H^{1}$ and BMO, Advances in Math., 22 (1976), pp. 269-277.

[8] P. Chen, X. T. Duong, J. Li, L. Song, And L. Yan, Carleson measures, BMO spaces and balayages associated to Schrödinger operators, Sci. China Math., 60 (2017), pp. 2077-2092.

[9] X. T. Duong, J. Li, Y. Ou, B. W. Wick, And D. YAng, Product BMO, little BMO and Riesz commutators in the Bessel setting, J. Geom. Anal., DOI: 10.1007/s12220-017-9920-2, (2017).

[10] X. T. Duong, J. LI, B. D. Wick, , AND D. YANG, Factorization for Hardy spaces and characterization for BMO spaces via commutators in the Bessel setting, Indiana Univ. Math. J., 66 (2017), pp. 1081-1106.

[11] X. T. DuONG AND L. YAN, Duality of Hardy and BMO spaces associated with operators with heat kernel bounds, J. Amer. Math. Soc., 18 (2005), pp. 943-973.

$[12]$ - New function spaces of BMO type, the John-Nirenberg inequality, interpolation, and applications, Comm. Pure Appl. Math., 58 (2005), pp. 1375-1420.

[13] X. T. Duong, L. Yan, and C. Zhang, On characterization of Poisson integrals of Schrödinger operators with BMO traces, J. Funct. Anal., 266 (2014), pp. 2053-2085. 
[14] J. Dziubański, G. Garrigós, T. Martínez, J. L. Torrea, and J. Zienkiewicz, BMO spaces related to Schrödinger operators with potentials satisfying a reverse Hölder inequality, Math. Z., 249 (2005), pp. 329-356.

[15] S. FridLI, Hardy spaces generated by an integrability condition, J. Approx. Theory, 113 (2001), pp. 91-109.

[16] J. B. Garnett, Bounded analytic functions, vol. 96 of Pure and Applied Mathematics, Academic Press, Inc., New York-London, 1981.

[17] J. B. Garnett and P. W. Jones, BMO from dyadic BMO, Pacific J. Math., 99 (1982), pp. 351-371.

[18] F. John and L. Nirenberg, On functions of bounded mean oscillation, Comm. Pure Appl. Math., 14 (1961), pp. $415-426$.

[19] N. N. Lebedev, Special functions and their applications, Dover Publications, Inc., New York, 1972. Revised edition, translated from the Russian and edited by Richard A. Silverman, Unabridged and corrected republication.

[20] J. Li And B. W. Wick, The two-weight inequality for the Poisson operator in the Bessel setting. Preprint 2017 arXiv:1707.07492.

[21] B. Muckenhoupt and E. M. Stein, Classical expansions and their relation to conjugate harmonic functions, Trans. Amer. Math. Soc., 118 (1965), pp. 17-92.

[22] A. Nowak and K. Stempak, Weighted estimates for the Hankel transform transplantation operator, Tohoku Math. J. (2), 58 (2006), pp. 277-301.

[23] —_ Potential operators associated with Hankel and Hankel-Dunkl transforms, J. Anal. Math., 131 (2017), pp. $277-321$.

[24] Z. W. Shen, $L^{p}$ estimates for Schrödinger operators with certain potentials, Ann. Inst. Fourier (Grenoble), 45 (1995), pp. 513-546.

[25] E. M. Stein, Harmonic analysis: real-variable methods, orthogonality, and oscillatory integrals, vol. 43 of Princeton Mathematical Series, Princeton University Press, Princeton, NJ, 1993.

[26] E. C. Titchmarsh, Hankel transforms, Proc. Camb. Phil. Soc., 21 (1923), pp. 463-473.

[27] A. UchiYama, A remark on Carleson's characterization of BMO, Proc. Amer. Math. Soc., 79 (1980), pp. 35-41.

[28] M. Villani, Riesz transforms associated to Bessel operators, Illinois J. Math., 52 (2008), pp. 77-89.

[29] J. M. Wilson, Green's theorem and balayage, Michigan Math. J., 35 (1988), pp. 21-27.

[30] D. YAng AND D. YAng, Real-variable characterizations of Hardy spaces associated with Bessel operators, Anal. Appl. (Singap.), 9 (2011), pp. 345-368.

Víctor Almeida, Jorge J. Betancor, Juan C. Fariña, Lourdes Rodríguez-Mesa

Departamento de Análisis Matemático, Universidad de la laguna,

Campus de Anchieta, Avda. Astrofísico Sánchez, s/n,

38721 la laguna (Sta. Cruz de Tenerife), Spain

E-mail address: valmeida@ull.es, jbetanco@ull.es, jcfarina@ull.es, lrguez@ull.es

Alejandro J. Castro

Department of Mathematics, NaZarbayev University,

010000 Astana, KaZAKhstan

E-mail address: alejandro.castilla@nu.edu.kz 